\title{
Reserve procurement and flexibility services in power systems with high renewable capacity: effects of integration on different market designs
}

\author{
Ruth Domínguez $^{1} \quad$ Giorgia Oggioni ${ }^{2} \quad$ Yves Smeers $^{3}$ \\ ${ }^{1}$ University of Castilla-La Mancha, Department of Electrical Engineering, Toledo, Spain. \\ ${ }^{2}$ University of Brescia, Department of Economics and Management, Brescia, Italy. \\ ${ }^{3}$ CORE, Université catholique de Louvain, Louvain-la-Neuve, Belgium.
}

\begin{abstract}
This paper investigates different reserve issues inspired by the European situation, focusing on both the moment when reserves are procured and the degree of coordination among Transmission System Operators (TSOs) in that procurement. We present three scheduling models formulated as stochastic programs that represent the day-ahead energy market, the reserve procurement, and the real-time balancing market in a renewable-dominated power system. Two of the proposed models are inspired by reserve procurement mechanisms currently applied in Europe, where reserves are committed either before (Model 1) or after (Model 2) the clearing of the day-ahead energy market. Then, we use as benchmark a third model in which energy and reserve capacity are co-optimized (Model 3). In all models, we consider the procurement of both conventional and upward/downward reserves. We also assess the impact of these organizations on market participants' remuneration and test the impact of cross-border constraints as those applying in the European power system. The case study is based on the IEEE 24-node RTS, considering the uncertainty in renewable power production and demand. Our results show that Model 1 is the least efficient market design as it leads to a misallocation of the available capacity, while Model 2 becomes as efficient as Model 3 when the TSOs procure reserve in a coordinated way. Finally, a coordinated procurement of reserves reduces the system operating costs in all models.
\end{abstract}

Keywords - Electricity market design and integration, Reserve procurement, Flexibility services, Stochastic programming, Renewable power production.

\section{Introduction}

\subsection{The European context and paper motivations}

The restructuring of the European electricity markets, started in the 1990s, has deeply changed the organization and the architecture of this sector. Directive 96/92/EC (first legislative package) ${ }^{1}$ was issued by the European Parliament and the Council to liberalize electricity markets and to create the Internal Electricity Market. This directive provided a few basic common principles in order to enforce a minimum market opening, define the non-discriminatory access to the grid, establish a transparent competition, and guarantee the demand supply in a sector that, before that, was vertically integrated and controlled by a sole entity operating in a monopolistic regime. These goals have been further enhanced by Directive 2003/54/EC (second legislative package) ${ }^{2}$ that enabled new electricity producers to enter in the wholesale markets and gave the possibility to consumers to choose their own electricity suppliers. Directive 2009/72/EC (third legislative package) ${ }^{3}$ and the related ENTSO-E Network Codes for electricity ${ }^{4}$ have been introduced with the aim of creating a single and coordinated European

\footnotetext{
${ }^{1}$ Directive 96/92/EC is available at https ://eur-lex.europa.eu/legal-content/EN/TXT/PDF/?uri=CELEX : 31996L0092\& from=EN

${ }^{2}$ Directive 2003/54/EC is available at https://eur-lex.europa.eu/resource.html?uri=cellar:caeb5f68-61fd-4ea8b3b5-00e692b1013c.0004.02/D0C_1\&f ormat=PDF

${ }^{3}$ Directive $2009 / 72 / \mathrm{E}$ is available at http://eur-lex.europa.eu/legal-content/EN/TXT/PDF/?uri=CELEX : 32009L0072\& from=en

${ }^{4}$ See ENTSO-E at https://electricity.network-codes.eu/network_codes /
} 
electricity market. This has as implication the integration of the day-ahead markets and the ancillary services represented by congestion management, reserve procurement, and balancing markets. The integration of all these services is becoming more challenging because of the increasing penetration of the intermittent generation technologies and the different organizations of the corresponding markets in the European countries. A fourth energy package (the so-called Winter package 2016) was introduced in November 2016. ${ }^{5}$ This text is still at an early stage in the legal process and is expected to be significantly modified before it becomes law. However, one can safely assume that the demand for more coordination will remain.

The current organization of the European electricity market is based on the separation of the energy and the ancillary services markets. This organization applies in each member state: Power Exchanges (PXs) clear the day-ahead electricity market taking into account a zonal representation of the network. Transmission System Operators (TSOs) procure reserve capacity, manage network congestion, and resolve residual imbalances in real time after an Intraday Market (IM) enables "Balancing Responsible Parties" (BRP) to manage some of these balances. This presence of several PXs and TSOs operating at national or subnational level obviously creates considerable coordination problems. The integration of the European day-ahead electricity markets has now become a reality thanks to the application of the Price Coupling of Regions (PCR) and the development of the Flow-Based Market Coupling approach. The PCR effectively coordinates day-ahead prices of energy by pricing "cross-border transmission capacities". The mechanism was developed at the initiative of several PXs but did not imply (at least so far) much harmonization among the national PXs. The "EUPHEMIA" algorithm that couples the regional markets also contributed to take care of national differences. ${ }^{6}$

In contrast with the realization of the day-ahead market, the way torwards the coordination of the intraday and the ancillary services markets remains long. A comprehensive study on the design elements of intraday and real-time markets of six European power markets is presented in Neuhoff et al. (2015). This paper highlights the different and the common elements in the design of these markets. For instance, as indicated in Section 2.4 of Neuhoff et al. (2015), "countries apply different rules for different reserve categories and may thus mandate different actors to procure the reserve". The differences appear in the reserve pricing structure, in the approach adopted for reserve pooling and procurement. In most of the European countries, reserves are procured by TSO monthly or even annually in advance their deployment as it happens, for example, in Belgium or in the Netherlands. Only few countries, like Italy or Spain, procure ancillary services once the day-ahead energy market is cleared. As observed by Baldick, ${ }^{7}$ "the preference by TSOs to procure reserves in advance is likely a manifestation of concerns about making sure there are enough reserves "on the day". However, ceteris paribus, the capacity for reserves will not change just because it is procured a week or a month in advance. Moreover, contracting monthly or yearly imposes a much greater risk that there will be outages between the time of contracting and the operating day." This reserve procurement mechanism was efficient in the past when power systems were mainly based on conventional technologies. However, the current configuration of the generation mix with a high share of wind and solar power makes electricity markets more unstable due to the stochasticity of renewable energy sources. In this context, a reserve allocation operated closer to real time and accounting for updated market information may be more efficient. The European Commission has recognized this in Article 32, Paragraph 2, item (b) of the guideline on Electricity Balancing, adopted on 23 November 2017 (see Commission Regulation, 2017b), where it is clearly stated that "the procurement process shall be performed on a short-term basis to the extent possible and where economically efficient." This is the reason why we try to develop a methodology for comparing the impact of a commitment of capacity to reserve taking place before and separately from the clearing of the day-ahead energy market to one where reserve capacity is selected after day-ahead clearing through an arbitrage with the capacity used for energy generation. This latter approach, which is effectively taking place in some European systems, is inspired by the joint procurement of energy and reserve in day-ahead operated by an Independent System Operator (ISO) in the US electricity markets. In addition, considering the decarbonization targets imposed by the European Commission through the Energy Roadmap 2050 (see European Commission, 2011) and the Winter package, which imply an increasing penetration of renewable sources in the electricity generation, the timing when reserves are procured may become important when reserve needs increase.

The European Commission has effectively recognized the importance of a coordinated procurement of reserves and an integrated balancing mechanism. It is evident that more harmonization is needed to facilitate coordination but the extent to which this will be achieved remains to be unknown. Several pilot initiatives have been set up at European level in order to facilitate the implementation of the Electricity Balancing Guideline. Among them, we recall TERRE (Trans European Replacement Reserve Exchange) and EXPLORE (European X-border project for Long term Real-time balancing Electricity market design). TERRE is a pilot project for cross-border Replacement

\footnotetext{
${ }^{5}$ See the European Commission package "Clean Energy for All Europeans" available at https://ec.europa.eu/energy/ en/topics/energy - strategy-and-energy - union/clean-energy-all-europeans

${ }^{6}$ See EPEXSPOT at https://ww .epexspot.com/en/market-coupling/pcr

${ }^{7}$ See Baldick R. (2017). Why doesn't the EU co-optimize the procurement of ancillary services with energy? available at http://fsr.eui.eu/doesnt-eu-co-optimize-procurement-ancillary-services-energy/
} 
Reserve (RR) exchanges. ${ }^{8}$ The aim of TERRE is to build an RR platform and to set up the European RR balancing energy market in order to create a harmonized playing fields for the market participants. It involves several TSOs, nine of them as participants and the five others (including ENTSO-E) as observers. The countries that officially members of the TERRE project are: France, Great Britain, Italy, Portugal, Spain, Switzerland, Czech Republic, Poland, and Romania (see Nationalgrid et al., 2017). ${ }^{9}$ In parallel, since the beginning of 2015, the TSOs of Austria, Belgium, Germany and the Netherlands have been studying the possibility of designing a common mFRR (manual Frequency Restoration Reserve) and aFRR (automatic Frequency Restoration Reserve) ${ }^{10}$ energy markets in the scope of the requirements of the draft guidelines of electricity balancing. The evolution of this initial project leads to the realization of two new programs: MARI (Manually Activated Reserves Initiative) for research on frequency restoration reserve with manual activation $(\mathrm{mFRR})^{11}$ and PICASSO (Platform for the International Coordination of the Automatic frequency restoration process and Stable System Operation) for exploring the integration of automatic frequency restoration reserves (aFRR) ${ }^{12}$ respectively launched in April and August, 2017. However, the European countries participating to all these projects have different ancillary services markets, imbalance pricing systems, and reserve procurement mechanisms.

Finally, and just to recall the importance of this internal electricity market objective, Newbery et al. (2016) evaluate the benefits of integrating European electricity markets through the coupling of inter-connectors to increase the efficiency of trading day-ahead, intra-day and balancing services at cross-border level. They estimate the economic gain of this market coordination at around 3.9 billion $€ / \mathrm{yr}$. All this legal activities show that reserves, their harmonization and the interaction of these reserves in the EU system may become a key issue for the European electricity market.

In this paper, we explore how reserve procurement in the European electricity market may suffers from both improper market design in individual countries (when the reserve procurement and energy market clearing take place at different times) and the interaction between the coordination of reserve procurement and the already coordinated day-ahead market. In doing this, we also take into account the uncertainty related to demand and renewable energy penetration.

\subsection{Literature review}

Numerous papers can be found in the technical literature addressing the issue of reserve requirements in power systems. We describe the most relevant contributions in the following.

Before the massive integration of intermittent renewable sources, the models proposed to determine the reserve capacity needs took into account the security requirements, as presented in Bouffard et al. (2005) and Wong and Fuller (2007). However, the increasing integration of renewable sources for the electricity generation has obliged to develop new mechanisms to optimally schedule energy and reserve capacity while ensuring the economical sustainability of the system.

The following papers deal with determining the reserve capacity needed to counteract the wind power uncertainty. Doherty and O'Malley (2005) propose a methodology to evaluate the reserve needs based on a probabilistic approach that takes into account the load and wind forecast errors and the probability of generator outages. Ortega-Vazquez and Kirschen (2009) conclude that the reserve requirements do not need to be significantly higher in power systems with high wind capacity. Papavasiliou et al. (2011) propose a scenario reduction technique on wind scenarios to determine the reserve requirements. In our paper, we consider the methods presented in OrtegaVazquez and Kirschen (2009) to determine the reserve capacity needs. However, differently from Ortega-Vazquez and Kirschen (2009) and the aforementioned papers, we focus more on the economic outcome of different reserve procurement mechanisms currently applied in Europe.

In order to simplify our analysis, we refer to stochastic programming theory (see Birge and Louveaux, 1997) and we systematically assume that the clearing of the day-ahead energy and real-time balancing markets can be represented by stochastic programming models that link the two markets. This approach, where day ahead is the first stage and real time balancing is the second stage, has been widely used in the technical literature (see Conejo et al., 2010) because it represents a useful stylized paradigm, even though it is implemented neither in the US nor in Europe. To the best of our knowledge, our paper is the first one that use stochastic programming to compare the efficiency of several market designs that differ for the mechanism adopted to schedule and procure reserves in renewable-dominated power systems. Other papers in the literature use two-stage stochastic programming models to investigate electricity markets in the presence of renewable energy sources penetration, such as Pritchard et al. (2010), Morales et al. (2012), and Zavala et al. (2017), but with different purposes. More precisely, the first two

\footnotetext{
${ }^{8}$ Replacement Reserves, in the new EU terminology, correspond to tertiary reserves in line with the Guideline on Electricity Balancing. See Commission Regulation (2017a).

${ }^{9}$ More details are available at https://www. entsoe.eu/network_codes/eb/terre/\#consultations

${ }^{10} \mathrm{mFRR}$ and aFRR, in the new EU terminology, correspond to the secondary reserves.

${ }^{11}$ For more details see https://www.entsoe.eu/network_codes/eb/mari/

${ }^{12}$ For more details see https://www.entsoe.eu/network_codes/eb/picasso/
} 
works address the problem of revenue adequacy and show that this is, in expectation, guaranteed in energy-only and energy and reserve co-optimization markets when the uncertainty of the wind power production is considered. They also highlight that, even though this is a general result, some exceptions are possibile, depending on the realizations of the uncertain parameters. On the other side, Zavala et al. (2017) prove that their formulation leads to bounded price distortions, zero uplifts in expectation, and day-ahead quantities similar to the corresponding real-time ones. In addition, as proved by Morales and Pineda (2017), the so-called arbitrage free property of finance is violated in this market representation. Note that this analysis is out of the scope of our paper.

Considering some applications to the European electricity market, we recall the following papers. Gebrekiros et al. (2015) present a reserve procurement approach comprising three decision stages: first, reserve providers determine their offers based on an opportunity cost method; second, the TSO sorts the offers under a merit order rule and determines the accepted ones; third, the day-ahead market is cleared considering the reserve capacity already scheduled. As a difference with our proposal, in this approach the balancing market is not considered, no economic analysis of the market participants is carried out, and no transmission capacity limits are considered.

Delikaraoglou et al. (2016) propose stochastic equilibrium problems to study the effects of different levels of spatio-temporal coordination in the clearing of regional electricity markets with significant stochastic generation. The results obtained from an illustrative numerical example show how much the social welfare reduces when there is lack of coordination. However, in this model no inter-temporal constraints are included (as, instead, we do consider) and the only uncertain parameter is the wind production.

Finally, Van den Bergh et al. (2017) investigate the value of inter-zonal coordination of reserve sizing, allocation and activation in the Central Western European electricity system. First, the zonal reserve requirements are determined as a function of the probabilistic density function of the wind and solar power forecast errors through a deterministic problem. Second, a unit commitment day-ahead energy market is solved considering the reserve capacity already fixed. Third, a real-time operating model is solved considering perfect information. No interchange capacity limitations are considered at the reserve sizing stage. Through the case study, they compare the costs obtained from different levels of coordination. In our proposal, we complete these analysis by comparing three market designs, i.e. co-optimization and no co-optimization of energy and reserve schedule and reserve scheduling before and after the day-ahead energy market.

\subsection{Paper objectives and contributions}

This paper deals with reserve procurement mechanisms in power systems with high renewable capacity and focuses on the following aspects:

1. The impact of the moment when reserves are scheduled;

2. The impact of the joint procurement of energy and reserves (co-optimization in the US parlance);

3. The effects of a geographically coordinated reserve procurement.

In order to assess the first item, we start by considering the market design applied in most of the European countries: energy and ancillary services markets are separated and reserves are scheduled before the clearing of the day-ahead energy market. We then compare this market configuration with another one also implemented in Europe, where day-ahead energy and ancillary services markets are still separated in day ahead, but reserves are committed after the clearing of the day-ahead energy market by arbitraging their value with the one of the capacity committed in the day-ahead energy market. This obviously implies a revision of the commitment of the capacities for the energy market and some compensation because of that revision. This alternative market organization is inspired by the Italian electricity system where the TSO operates the ancillary services market, after the clearing of the day-ahead market, following a central dispatch approach that co-optimizes the re-dispatch, the reserve procurement, and the energy balancing. ${ }^{13}$ This process is affected by a very specific restriction: the re-dispatching activities conducted by the TSO are constrained to maintain the net commercial transactions between zones as determined in the clearing of the day-ahead energy market. In other words, the cross-border flows among zones cannot be modified from those obtained in the day-ahead energy market by PXs. It reflects the importance given to the day-ahead market in the European organization, where the real time is a technical market aimed at keeping the day-ahead outcome feasible. This view is probably best expressed in Article 3, Paragraph 2, item (e) of the Balancing Electricity Guideline (see Commission Regulation, 2017b). ${ }^{14}$ This movement towards co-optimization in the Italian ancillary services market could suggest a possible future evolution of the European

\footnotetext{
${ }^{13}$ See the grid code published by Terna, the Italian TSO, at http://www.terna.it/en-gb/sistemaelettrico/ codicedirete. aspx

${ }^{14}$ Article 3, Paragraph 2, item (e) of the guideline on Electricity Balancing states that "...relevant regulatory authorities and systems operators shall: ... (e) ensure that the development of the forward, day ahead and intraday markets are not compromised."
} 
electricity market; it indeed accomplishes the requisites of Article 32 of the guideline on Electricity Balancing stating that reserve procurement shall be performed close to real time. ${ }^{15}$

This implicit recourse to co-optimization in the comparison of the ex-ante and ex-post (with respect to day ahead) commitment of reserve suggests a third model where capacities are simultaneously committed to energy and reserve. This is directly inspired by the US market design. The combination of these three different market designs (ex-ante, ex-post, and simultaneous commitment) provides the basis for the analysis of the second item.

On the other side, the third item deals with the standard problem of coordination of policies among different zones. We compare the three aforementioned market configurations, assuming that the procurement of reserve is done in autarky in different zones or integrated among these zones. This is done by decomposing a IEEE problem in different zones and applying the three market designs on these two situations.

Therefore, the contributions of this paper can be summarized as follows:

1) We propose stylized models of two market designs currently implemented in European power systems to schedule energy and reserve capacity that differ from the time in which reserve capacity needs are procured. Additionally, we compare these European market designs with the joint procurement of energy and reserves applied in the US. In all models, we consider a zonal reserve procurement and the uncertainty related to the renewable power production and the demand.

2) We analyze and compare the numerical results obtained from the three models considering two aspects: the timing of the reserve procurement and different levels of TSO coordination in the reserve procurement.

3) In addition, we provide some insights on the application of these different designs on the incomes of the market players. The innovative aspect is that we use the duality principles of linear programming to compute the consumers' benefit, the ISO/TSOs' remuneration, and the generating units' profits. In the generating units' profits we separate between those resulting from providing energy and reserve capacity, and those related to ramping flexibility services.

4) Our analysis shows the important impact of the market design and the lack of coordination on the efficiency and sustainability of power systems. The numerical analysis highlights how choices of market designs and idiosyncratic constraints (inspired by existing EU practices) can result in unnecessary costs and unserved demand. This outcome is obtained notwithstanding the optimistic assumptions made on several aspects of the market.

\subsection{Paper structure}

The rest of the paper is structured as follows. Section 2 describes the assumptions underpinning the three models as well as their mathematical formulations. Section 3 describes the input data of the case study, while the obtained results are analyzed in Section 5. Section 6 concludes with some final remarks. Additional material is reported in the appendices. In particular, A reports the notation used in the models, while B explains the method for computing the market players' benefits. C and D provide a detailed description of the input data used in the case study and of the scenario generation, respectively.

\section{Model description}

We present three models that embed a day-ahead energy market, the reserve procurement (before or after the day-ahead market), and a balancing market. The latter is cleared after the revelation of renewable generation and demand. In all models, we account for perfect competition, namely all market players are price takers. In this section, the common assumptions considered to model the market designs under study and the proposed formulations are provided.

\subsection{Common assumptions}

The common assumptions are listed below:

1) Spatial granularity of the markets. In order to facilitate the comparison of the results, we adopt the same nodal granularity both in the day-ahead and in the balancing markets in all models. The nodal system is in force in the US, but not in Europe. Assuming nodal pricing everywhere eliminates any discussion of idiosyncratic congestion effect and enables concentrating on the reserve issue.

2) Spatial granularity of reserve procurement. Reserves are procured on a zonal basis. For this reason, nodes are grouped into zones that are interpreted as, for instance, the countries in the European electricity market. This holds for all considered models.

\footnotetext{
${ }^{15}$ Article 32, Paragraph 2, item (b) of the guideline on Electricity Balancing indicates that "the procurement process shall be performed on a short-term basis to the extent possible and where economically efficient;"
} 
3) Degree of coordination in reserve procurement. We assume two situations: independent operation of regional TSOs, and an upper organization of TSOs, namely CORESO, ${ }^{16}$ that can globally operate the reserve procurement and the balancing market. In our analysis, we refer to a "Coordinated reserve procurement" when this supranational TSO procures reserves and dispatches the balancing market, while "Not-coordinated reserve procurement" refers to the situation where TSOs work in an independent way.

4) Reserve types. We consider conventional and upward/downward reserves. In order to make the three models comparable, we impose a minimum level of upward and downward reserve capacity in each hour. We provide more details on reserves in Section 2.2.

5) Generating units. Power units are subdivided into dispatchable (nuclear, coal, combined cycle gas turbine (CCGT)) and stochastic (wind and solar photovoltaic (PV)) units. The proposed models are constructed over a multi-period horizon and include inter-temporal (ramping) constraints on dispatchable units. Dispatchable units are further classified into qualified and not-qualified depending on whether they can provide reserves or not. The criterium to be eligible for reserves differs among the power systems. For the sake of simplicity, we assume that coal and CCGT power plants are qualified units and provide reserves. To avoid non-linearities, we do not include unit commitment variables. As to stochastic units, we consider that their offers in day ahead are limited to their capacity. Power spillage is allowed in real time to maintain the system feasibility.

6) Unserved demand. The models contain representations of demand curtailment both in day-ahead and in real-time markets. Demand curtailment arises when generation does not suffice to cover the total demand of electricity (unserved demand).

7) Demand Side Management (DSM). We also consider DSM in our models, as an additional reserve service that demand can provide in the balancing market upon request of the TSO. This is done by increasing or decreasing consumer's demand compared to day-ahead announcement, subject to a deviation margin that is supposed to be relatively low in comparison with the predicted consumption. We consider that the demand deviation cost is higher than that of deploying upward reserve by qualified units. Moreover, the unserved demand cost is assumed to be significantly higher than the demand deviation cost in DSM so that there will be involuntary unserved demand only in exceptional situations.

8) Uncertainty. Demand level and renewable power production are the uncertain parameters and their values are represented by a set of scenarios. A scenario specifies the demand level and the available wind and solar power in each hour and in each node of the network. The construction of scenarios is described in Appendix D (see Supplementary Material).

9) Balancing market. As stated in Section 1.2, we assume that balancing market is perfectly coordinated in all models and under all reserve coordination assumptions.

\subsection{Reserve types and classification}

Primary, secondary and tertiary controls are used to restore the balance between generation and load whenever disturbed. The primary reserve has to the role of stabilizing the system frequency and has to respond to frequency signals in a short notice, typically within 5-10 seconds. It can ramp up to its full output in 30-60 seconds. After primary control stabilizes the frequency, it is replaced by secondary control that relieves the primary reserves so that they can return to their normal condition. The secondary reserves are automatically controlled by the system operator and must usually respond in 30 seconds. After the frequency has been completely restored, tertiary reserves are meant to free up secondary reserves, to make the system able to respond to the next contingency. The operation mode is manually and it typically needs to respond in 15 minutes.

Depending on the considered market, this reserve classification is identified in different ways. Table 1 shows the nomenclature currently adopted in the US (see Chen et al., 2014; Cramton, 2017; Ela et al. 2011 and FloresEspino, 2016) and the European (Commission Regulation, 2017a) markets and the respective correspondences (see Baldick, 2017).

\begin{tabular}{|l|l|l|l|}
\hline Reserve classification & Activation time & US reserve classification & EU reserve classification \\
\hline \hline Primary Reserves & up to $30 \mathrm{~s}$ & Regulating Reserves & $\begin{array}{l}\text { Frequency Containment } \\
\text { Reserves (FCR) }\end{array}$ \\
\hline Secondary Reserves & from $30 \mathrm{~s}$ to $15 \mathrm{~m}$ & Spinning Contingency Reserves & $\begin{array}{l}\text { automatic Frequency } \\
\text { Restoration Reserves (aFRR) }\end{array}$ \\
\hline Tertiary Reserves & from $15 \mathrm{~m}$ to $120 \mathrm{~m}$ & $\begin{array}{l}\text { Non-spinning or Supplemental } \\
\text { Contingency Reserves }\end{array}$ & Replacement Reserves (RR) \\
\hline
\end{tabular}

Table 1: Reserve classifications

\footnotetext{
${ }^{16}$ See http://www . coreso.eu/
} 
In our analysis, we assume that reserves are split into upward/downward and conventional reserves. Upward/downward reserves are used to counteract the real time deviations due to forecast errors in the demand and the renewable stochastic production. Conventional reserves respond to contingencies and are ruled by a standard $\mathrm{N}-1$ criterion in day ahead, even though we assume no contingency event in the real time operation. Considering the European jargon, our upward/downward reserves correspond to the automatic frequency restoration reserves, while what we denote as conventional reserves represent the replacement reserves.

We assume that both types of reserves are zonally procured by TSOs/CORESO on a hourly basis in order to ensure the reliability of the system (this type of reserves is defined as ramping reserve in Milligan et al., 2010). The zones are defined according to the area controlled by each TSO. Flexible dispatchable units such as coal and CCGT units provide this reserve capacity. We assume that offline CCGT and coal units can provide upward and conventional reserve capacity. Their power output is limited by their ramp capability. Following AREVA T\&D Technology Centre (2006) and the discussion in Ortega-Vazquez and Kirschen (2009), we assume that upward and downward reserves are determined as a function of the standard deviation of the forecast error distributions of both the demand level and the stochastic production. Note that the forecast errors are observed in real time, and hence, in our models are associated with the real-time scenarios. This approach is comparable to those used by the TSOs in reality (see Milligan et al., 2010). Finally, we do not consider a direct cost for scheduling reserve capacity in day ahead. The cost of providing reserve services is evaluated in the balancing market in terms of the energy supplied to counteract deviations.

\section{$2.3 \quad$ Model formulations}

This section provides the mathematical formulations of the proposed models. The symbols used in the following formulations are described in Appendix A.

\subsubsection{Model 1: Reserves scheduled before the day-ahead energy market}

Model 1 is inspired by some common features of various European market designs of Central-Western Europe, where TSOs procure reserves, offered by qualified units, before the PXs clear the day-ahead energy markets (Neuhoff et al., 2015). Note that, in the European context, national PXs co-exist with PCR that plays the role of a central clearing house. Afterwords, the PXs/PCR clears the day-ahead energy market to which qualified units participate, but their bids are restricted to the part of the capacity that has not been committed as reserves. Finally, TSOs operate the balancing market.

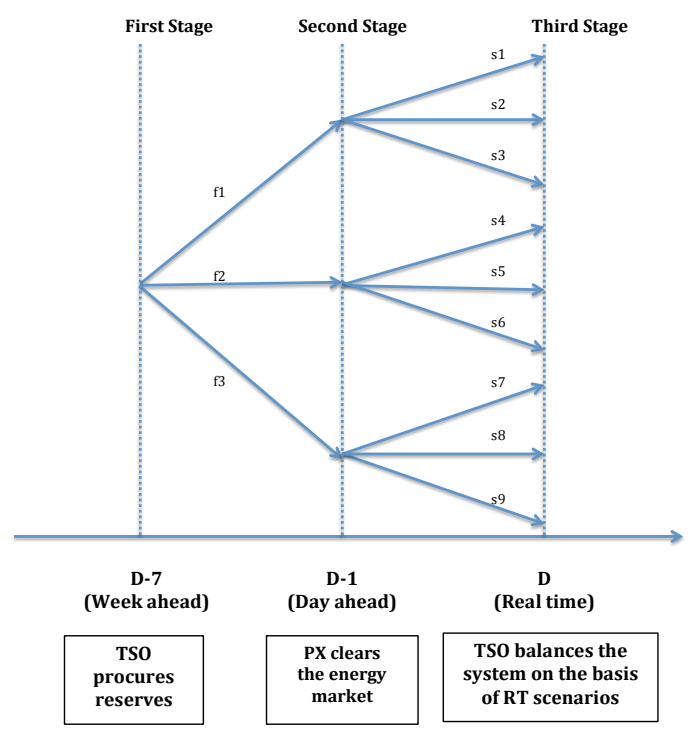

Figure 1: Decision-making process of Model 1

This market configuration is translated into a three-stage stochastic model that is structured as follows: reserve procurement takes place in the first stage (e.g. one week ahead or D-7); in the second stage, the day-ahead energy market clears on the basis of demand and renewable production forecasts (D-1); and the balancing market, which is the third stage, takes place in real time (D) on the basis of renewable and demand realizations. Since upward/downward reserves are committed before renewable production and demand forecasts are revealed (e.g. 
in D-7), the TSOs determine the minimum reserve capacity on the basis of the largest possible reserve that can be required given the renewable production and the demand forecasts. In other words, reserve procurement is based on the worst realization of demand level and stochastic unit production. Obviously, qualified units committed to provide reserves in D-7 can bid energy in D-1 only up to their residual capacity. The decision-making process is depicted in Figure 1.

The PXs/PCR and the TSOs/CORESO operate the energy, the reserve and the balancing markets by minimizing the total expected costs, as indicated in the mathematical formulation of the model presented below:

$$
\begin{aligned}
& \operatorname{Min} \sum_{t=1}^{\mathrm{N}_{\mathrm{T}}} \sum_{f=1}^{\mathrm{N}_{\mathrm{F}}} \xi_{f}\left[\sum_{g \in\left\{G(g, n), \Omega^{\mathrm{Di}}\right\}} C_{g}^{\mathrm{G}} p_{g t f}^{\mathrm{DA}}+\sum_{j=1}^{\mathrm{N}_{\mathrm{J}}} C^{\mathrm{US}} d_{j t f}^{\mathrm{US} \mathrm{DA}}\right]+\sum_{t=1}^{\mathrm{N}_{\mathrm{T}}} \sum_{s=1}^{\mathrm{N}_{\mathrm{S}}} \sum_{g \in \Omega^{\mathrm{Q}}} \xi_{s}\left[C_{g t}^{\mathrm{DUR}} r_{g t s}^{\mathrm{U}}-C_{g t}^{\mathrm{DDR}} r_{g t s}^{\mathrm{D}}\right]+ \\
& +\sum_{t=1}^{\mathrm{N}_{\mathrm{T}}} \sum_{s=1}^{\mathrm{N}_{\mathrm{S}}} \sum_{j=1}^{\mathrm{N}_{\mathrm{J}}} \xi_{s}\left[C_{j}^{\mathrm{D}}\left(\Delta d_{j t s}^{\mathrm{U}}+\Delta d_{j t s}^{\mathrm{D}}\right)+C^{\mathrm{US}} d_{j t s}^{\mathrm{US} \mathrm{RT}}\right]
\end{aligned}
$$

subject to

$$
\begin{aligned}
& \left.\sum_{g \in\left\{Z G(g, z), \Omega^{\mathrm{Q}}\right\}} R_{g t}^{\mathrm{D}} \geq K^{\mathrm{D}} \sqrt{\max _{f}\left\{\sum_{j \in Z D(j, z)}\left(\sigma_{j t f}^{\mathrm{D}}\right)^{2}\right\}+\max _{f}\left\{\sum_{g \in\left\{Z G(g, z), \Omega^{\mathrm{St}}\right\}}\left(\sigma_{g t f}^{\mathrm{St}}\right)^{2}\right.}\right\} \forall z, \forall t \quad\left(\eta_{z t}^{D}\right) \\
& \left.\sum_{g \in\left\{Z G(g, z), \Omega^{\mathrm{Q}}\right\}} R_{g t}^{\mathrm{U}} \geq K^{\mathrm{U}} \sqrt{\max _{f}\left\{\sum_{j \in Z D(j, z)}\left(\sigma_{j t f}^{\mathrm{D}}\right)^{2}\right\}+\max _{f}\left\{\sum_{g \in\left\{Z G(g, z), \Omega^{\mathrm{St}}\right\}}\left(\sigma_{g t f}^{\mathrm{St}}\right)^{2}\right.}\right\} \quad \forall z, \forall t \quad\left(\eta_{z t}^{U}\right) \\
& \sum_{g \in\left\{Z G(g, z), \Omega^{\mathrm{Q}}\right\}} R_{g t}^{\mathrm{C}} \geq \max _{g \in\left\{Z G(g, z), \Omega^{\mathrm{Di}}\right\}}\left\{P_{g}^{\mathrm{Gmax}}\right\}, \quad \forall z, \forall t \quad\left(\eta_{z t}^{C}\right) \\
& \sum_{g \in G(g, n)} p_{g t f}^{\mathrm{DA}}-\sum_{j \in D(j, n)}\left(D_{j t f}^{\mathrm{F}}-d_{j t f}^{\mathrm{USA}}\right)-\sum_{l \mid O(l)=n} p_{l t f}^{\mathrm{LDA}}+\sum_{l \mid D(l)=n} p_{l t f}^{\mathrm{LDA}}=0, \quad \forall n, \forall t, \forall f \quad\left(\lambda_{n t f}^{\mathrm{DA}}\right) \\
& p_{l t f}^{\mathrm{LDA}}=B_{l}\left(\theta_{O(l), t f}^{\mathrm{DA}}-\theta_{D(l), t f}^{\mathrm{DA}}\right), \quad \forall l, \forall t, \forall f \quad\left(\rho_{l t f}^{\mathrm{p}}\right) \\
& -P_{l}^{\mathrm{Lmax}} \leq p_{l t f}^{\mathrm{LDA}} \leq P_{l}^{\mathrm{Lmax}}, \quad \forall l, \forall t, \forall f \quad\left(\rho_{l t f}^{\min }, \rho_{l t f}^{\max }\right) \\
& -\pi \leq \theta_{n t f}^{\mathrm{DA}} \leq \pi, \quad \forall n, \forall t, \forall f \quad\left(\rho_{n t f}^{\pi, \min }, \rho_{n t f}^{\pi, \max }\right) \\
& \theta_{n t f}^{\mathrm{DA}}=0, \quad n=r e f, \forall t, \forall f \quad\left(\rho_{n t f}^{0}\right) \\
& 0 \leq d_{j t f}^{\mathrm{US} \mathrm{DA}} \leq D_{j t f}^{\mathrm{F}}, \quad \forall j, \forall t, \forall f \quad\left(\psi_{j t f}\right) \\
& 0 \leq p_{g t f}^{\mathrm{DA}} \leq P_{g}^{\mathrm{Gmax}}, \quad \forall g \in \Omega^{\mathrm{nQ}}, \Omega^{\mathrm{St}}, \forall t, \forall f \quad\left(\mu_{g t f}\right) \\
& p_{g t f}^{\mathrm{DA}}-p_{g, t-1, f}^{\mathrm{DA}} \leq \operatorname{ramp}_{g}^{\mathrm{up}}, \quad \forall g \in \Omega^{\mathrm{nQ}}, \forall t, \forall f \quad\left(\mu_{g t f}^{\mathrm{rU}}\right) \\
& p_{g, t-1, f}^{\mathrm{DA}}-p_{g, t, f}^{\mathrm{DA}} \leq \operatorname{ramp}_{g}^{\mathrm{do}}, \quad \forall g \in \Omega^{\mathrm{nQ}}, \forall t, \forall f \quad\left(\mu_{g t f}^{\mathrm{rD}}\right) \\
& p_{g t f}^{\mathrm{DA}}+R_{g t}^{\mathrm{U}}+R_{g t}^{\mathrm{C}} \leq P_{g}^{\mathrm{Gmax}}, \quad \forall g \in \Omega^{\mathrm{Q}}, \forall t, \forall f \quad\left(\kappa_{g t f}^{\mathrm{U}}\right) \\
& p_{g t f}^{\mathrm{DA}}-R_{g t}^{\mathrm{D}} \geq 0, \quad \forall g \in \Omega^{\mathrm{Q}}, \forall t, \forall f \quad\left(\kappa_{g t f}^{\mathrm{D}}\right) \\
& \left(p_{g t f}^{\mathrm{DA}}+R_{g t}^{\mathrm{U}}+R_{g t}^{\mathrm{C}}\right)-\left(p_{g, t-1, f}^{\mathrm{DA}}-R_{g, t-1}^{\mathrm{D}}\right) \leq \operatorname{ramp}_{g}^{\mathrm{up}}, \quad \forall g \in \Omega^{\mathrm{Q}}, \forall t, \forall f \quad\left(\kappa_{g t f}^{r U}\right) \\
& \left(p_{g, t-1, f}^{\mathrm{DA}}+R_{g, t-1}^{\mathrm{U}}+R_{g, t-1}^{\mathrm{C}}\right)-\left(p_{g t f}^{\mathrm{DA}}-R_{g t}^{\mathrm{D}}\right) \leq \operatorname{ramp}_{g}^{\mathrm{do}}, \quad \forall g \in \Omega^{\mathrm{Q}}, \forall t, \forall f \quad\left(\kappa_{g t f}^{r D}\right) \\
& \sum_{g \in\left\{G(g, n), \Omega^{\mathrm{Q}}\right\}}\left(r_{g t s}^{\mathrm{U}}-r_{g t s}^{\mathrm{D}}\right)+\sum_{g \in\left\{G(g, n), \Omega^{\mathrm{St}}\right\}}\left(p_{g t s}^{\mathrm{G}}-p_{g t f}^{\mathrm{DA}}\right)+ \\
& -\sum_{j \in D(j, n)}\left(D_{j t s}^{\mathrm{RT}}-D_{j t f}^{\mathrm{F}}+\Delta d_{j t s}^{\mathrm{U}}-\Delta d_{j t s}^{\mathrm{D}}-d_{j t s}^{\mathrm{US}}\right)+ \\
& -\sum_{l \mid O(l)=n}\left(p_{l t s}^{\mathrm{LRT}}-p_{l t f}^{\mathrm{LDA}}\right)+\sum_{l \mid D(l)=n}\left(p_{l t s}^{\mathrm{LRT}}-p_{l t f}^{\mathrm{LDA}}\right)=0, \quad \forall n, \forall t, \forall f, \forall s \in \Omega_{f} \quad\left(\lambda_{n t s}^{\mathrm{RT}}\right) \\
& p_{l t s}^{\mathrm{LRT}}=B_{l}\left(\theta_{O(l), t s}^{\mathrm{RT}}-\theta_{D(l), t s}^{\mathrm{RT}}\right), \quad \forall l, \forall t, \forall s \quad\left(\gamma_{l t s}^{\mathrm{p}}\right) \\
& -P_{l}^{\mathrm{Lmax}} \leq p_{l t s}^{\mathrm{LRT}} \leq P_{l}^{\mathrm{Lmax}}, \quad \forall l, \forall t, \forall s \quad\left(\gamma_{l t s}^{\min }, \gamma_{l t s}^{\max }\right) \\
& -\pi \leq \theta_{n t s}^{\mathrm{RT}} \leq \pi, \quad \forall n, \forall t, \forall s \quad\left(\gamma_{n t s}^{\pi, \min }, \gamma_{n t s}^{\pi, \max }\right) \\
& \theta_{n t s}^{\mathrm{RT}}=0, \quad n=r e f, \forall t, \forall s \quad\left(\gamma_{n t s}^{0}\right)
\end{aligned}
$$




$$
\begin{aligned}
& 0 \leq r_{g t s}^{\mathrm{U}} \leq R_{g t}^{\mathrm{U}}, \quad \forall g \in \Omega^{\mathrm{Q}}, \forall t, \forall s \quad\left(\delta_{g t s}^{\mathrm{U}}\right) \\
& 0 \leq r_{g t s}^{\mathrm{D}} \leq R_{g t}^{\mathrm{D}}, \quad \forall g \in \Omega^{\mathrm{Q}}, \quad \forall t, \forall s \quad\left(\delta_{g t s}^{\mathrm{D}}\right) \\
& 0 \leq p_{g t s}^{\mathrm{G}} \leq P_{g t s}^{\mathrm{RT}}, \quad \forall g \in \Omega^{\mathrm{St}}, \quad \forall t, \forall s \quad\left(\tau_{g t s}\right) \\
& D_{j t s}^{\mathrm{RT}}+\Delta d_{j t s}^{\mathrm{U}}-\Delta d_{j t s}^{\mathrm{D}}-d_{j t s}^{\mathrm{US}} \geq 0, \quad \forall j, \forall t, \forall s \quad\left(\psi_{j t s}^{1}\right) \\
& 0 \leq \Delta d_{j t s}^{\mathrm{U}} \leq \Delta d_{j t}^{\max }, \quad \forall j, \forall t, \forall s \quad\left(\psi_{j t s}^{2}\right) \\
& 0 \leq \Delta d_{j t s}^{\mathrm{D}} \leq \Delta d_{j t}^{\max }, \quad \forall j, \forall t, \forall s \quad\left(\psi_{j t s}^{3}\right)
\end{aligned}
$$

The objective function (1) represents the total expected cost of operating the power system which has to be minimized. The first line of expression (1) represents the total expected cost of scheduling energy in the day-ahead market and the cost of the possible unserved demand for the set of day-ahead forecasts. The second line corresponds to the expected (net) cost of the reserves deployed by qualified units in real time. The last line represents the expected cost of the DSM and the unserved demand in the balancing market.

Constraints (2)-(4) refer to a week ahead (first stage), constraints (5)-(17) describe the day-ahead energy market (second stage), while (18)-(28) represent the real-time constraints (third stage).

In particular, constraints (2)-(4) impose the minimum zonal reserve needs in each period one week ahead. Specifically, constraints (2)-(3) enforce the upward/downward reserve requirements on the basis of the maximal standard deviation of the error distributions in the real-time scenarios in each day-ahead forecast. Parameters $\sigma_{j t}^{\mathrm{D}}$ and $\sigma_{g t}^{\mathrm{St}}$ are the standard deviations of the distribution of the forecast error of real-time scenarios, while $K^{\mathrm{D}}$ and $K^{\mathrm{U}}$ are security factors accounting for the spanning time between the predictions and the actual realization (see Ortega-Vazquez and Kirschen, 2009). Constraint (4) enforces the zonal scheduling of reserve capacity for dealing with contingencies.

The energy balance in the day-ahead energy market (second stage) is defined through (5) per each node, forecast, and period. Constraints (6)-(9) define the power flow through transmission lines in the day-ahead market and enforce the capacity limits of the lines. Constraint (9) set the voltage angle to zero in the reference node $(n=r e f)$. Constraint (10) limits the unserved demand to the forecast demand in each period. Constraint (11) limit the power output of not-qualified and stochastic units, while constraints (12)-(13) define the ramping limits of not-qualified units. Constraints (14)-(17) enforce capacity and ramping limits of qualified units considering the scheduled upward/downward and conventional reserves. Ramping constraints of qualified units are imposed on the reserve capacities (day-ahead market) to take into account the share of capacity used to meet the conventional reserve requirement, even though we assume that there is no contingency in the real-time operation. The idea is to have a surrogate of ramping products such as those found in US markets, following the principle that the dayahead market should plan for ramping requirements to some extent. This is done by using two sets of constraints: first, constraints (2) and (3) model the hourly reserve requirement related to the uncertain parameters (renewable energy production and demand); second, constraints (16) and (17) derive from cross-period requirements. These constraints do not add up: the reserve requirement must satisfy constraints that represent two views, but the model determines which constraints are binding.

Finally, constraints (18)-(28) define the operation of the balancing market. Constraint (18) represents the real-time energy balances in each node, each period, each day-ahead forecast scenario, and each real-time scenario generated from that day-ahead forecast scenario. At this stage, reserves are deployed to counteract the forecast errors of demand and renewable production respect to the day-ahead schedule. Constraints (19)-(22) limit the power flow through transmission lines in the same way as in the day-ahead market. Constraints (23) and (24) limit the deployed upward and downward reserves to what scheduled in day ahead. Constraint (25) limits the power generated in real time by wind/solar units as a function of the actual available power in each scenario. This constraint implicitly allows for wind spillage. Constraints (26)-(28) impose limits to the upward and downward DSM considering the actual demand level in each node, period, and scenario.

\subsubsection{Model 2: Reserves scheduled after the day-ahead energy market}

Model 2 describes a market where reserve needs are determined by the TSOs after the clearing of the dayahead energy market, as for the Italian electricity market. Model 2 is formulated via two optimization problems that describe the PXs/PCR and TSOs/CORESO's activities, respectively. Figure 2 depicts the decision-making process adopted in this market design.

First, the PXs/PCR clears the day-ahead energy market without any consideration for reserve requirements. This is modeled through a deterministic problem and represents a simplified version of EUPHEMIA, which clears the energy market without considering reserve constraints (or by considering them in some way that is not defined). All dispatchable units participate to this market and qualified units have no bidding limit. 


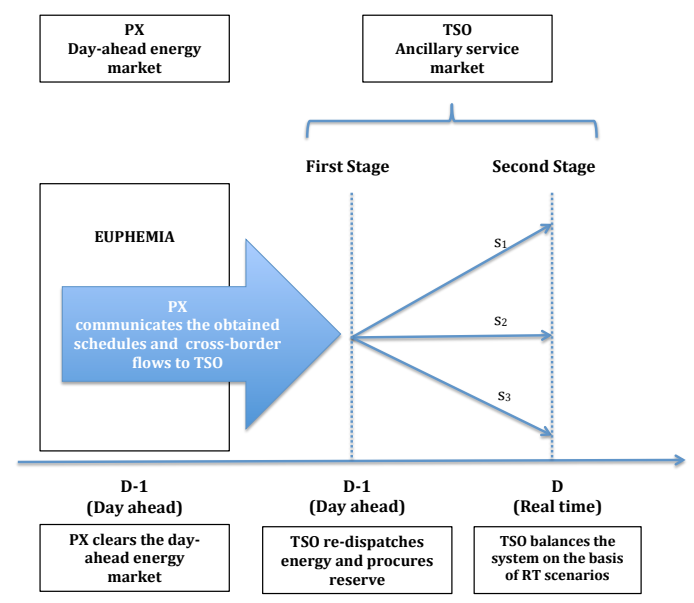

Figure 2: Decision-making process of Model 2

After the closure of the day-ahead energy market, the PXs/PCR communicates the obtained schedules for generating units and the cross-border flows to the TSOs/CORESO, which conducts the second process. In this second process, the TSOs/CORESO redefines the scheduling of qualified and not-qualified units (if needed) coming from the day-ahead energy market in order to satisfy the reserve requirements. Therefore, we assume that the TSOs/CORESO has the possibility to completely re-dispatch generating units. This second process is modeled through a two-stage stochastic program, in which the first stage represents the day-ahead rescheduling operated by the TSOs/CORESO, and the second stage represents the balancing market. The rationale for adopting this representation is that it corresponds to a real situation inside some European electricity markets: EUPHEMIA solves a pure day-ahead energy market without considering an endogenisation of reserve and then the TSOs procure reserve needs. Since the TSOs/CORESO operation takes place after the clearing of the day-ahead energy market, one could argue that an updated set of forecast scenarios is available to TSOs/CORESO compared to those used in day-ahead. This is what happens in the reality. However, in order to make results strictly comparable among all models, we neglect this aspect and assume that the same scenarios are available to PXs/PCR and TSOs/CORESO.

Moreover, as explained in Section 1.3, the re-dispatching activities conducted by the TSOs/CORESO are constrained to maintain the net commercial transactions between zones determined in the clearing of the dayahead energy market. This requirement is added to the TSOs/CORESO's model. Note that this may be a challenge in the future. Suppose for a moment a perfect coordination in the reserve procurement within the market design represented in this Model 2. A direct consequence of this organization is that the TSOs/CORESO either modifies the day-ahead outcome to conduct balancing or operates balancing in some sort of counter-trading model in order to keep day-ahead transactions unchanged. The mathematical formulations of the problems describing this market organization are provided below.

\subsubsection{PXs/PCR: day-ahead energy market}

This model represents the day-ahead energy market cleared by the PXs/PCR, which seeks to minimize the costs of energy production and unserved demand (29) subject to constraints (30)-(39) as follows:

$\operatorname{Min} \sum_{t=1}^{\mathrm{N}_{\mathrm{T}}}\left(\sum_{g \in\left\{G(g, n), \Omega^{\mathrm{Di}}\right\}} C_{g}^{\mathrm{G}} p_{g t}^{\mathrm{DA}}+\sum_{j=1}^{\mathrm{N}_{\mathrm{J}}} C^{\mathrm{US}} d_{j t}^{\mathrm{US}}\right)$

subject to

$\sum_{g \in\{G(g, n)\}} p_{g t}^{\mathrm{DA}}-\sum_{j \in D(j, n)}\left(D_{j t}^{\mathrm{F}}-d_{j t}^{\mathrm{US}}\right)-\sum_{l \mid O(l)=n} p_{l t}^{\mathrm{LDA}}+\sum_{l \mid D(l)=n} p_{l t}^{\mathrm{LDA}}=0, \quad \forall n, \forall t \quad\left(\lambda_{n t}^{\mathrm{DA}}\right)$ 


$$
\begin{aligned}
& 0 \leq p_{g t}^{\mathrm{DA}} \leq P_{g}^{F}, \quad \forall g \in \Omega^{\mathrm{St}}, \forall t, \quad\left(\mu_{g t}^{\mathrm{St}}\right) \\
& 0 \leq p_{g t}^{\mathrm{DA}} \leq P_{g t}^{\mathrm{Gmax}}, \quad \forall g \in \Omega^{\mathrm{Di}}, \forall t, \quad\left(\mu_{g t}\right) \\
& p_{g t}^{\mathrm{DA}}-p_{g, t-1}^{\mathrm{DA}} \leq \operatorname{ramp}_{g}^{\mathrm{up}}, \quad \forall g \in \Omega^{\mathrm{Di}}, \forall t \quad\left(\kappa_{g t}^{1}\right) \\
& p_{g, t-1}^{\mathrm{DA}}-p_{g t}^{\mathrm{DA}} \leq \operatorname{ramp}_{g}^{\mathrm{do}}, \quad \forall g \in \Omega^{\mathrm{Di}}, \forall h \quad\left(\kappa_{g t}^{2}\right) \\
& p_{l t}^{\mathrm{LDA}}=B_{l}\left(\theta_{O(l), t}^{\mathrm{DA}}-\theta_{D(l), t}^{\mathrm{DA}}, \quad \forall l, \forall t \quad\left(\rho_{l t}^{\mathrm{D}}\right)\right. \\
& -P_{l}^{\mathrm{Lmax}} \leq p_{l t}^{\mathrm{LDA}} \leq P_{l}^{\mathrm{Lmax}}, \quad \forall l, \forall t \quad\left(\rho_{l t}^{\min }, \rho_{l t}^{\max }\right) \\
& -\pi \leq \theta_{n t}^{\mathrm{DA}} \leq \pi, \quad \forall n, \forall t \quad\left(\rho_{n t}^{\pi, \min }, \rho_{n t}^{\pi, \max }\right) \\
& \theta_{n t}^{\mathrm{DA}}=0, \quad n=r e f, \forall t \quad\left(\rho_{n t}^{0}\right) \\
& 0 \leq d_{j t}^{\mathrm{US}} \leq D_{j t}^{\mathrm{DA}}, \quad \forall j, \forall t \quad\left(\psi_{j t}\right)
\end{aligned}
$$

Constraint (30) represents the energy balance. We assume that the power scheduled by renewable generating units is restricted by the corresponding forecasts as indicated in constraint (31). Constraints (32)-(34) indicate the production capacity and the ramping limits imposed on dispatchable units. In this model, capacity represents the unique restriction imposed on the bids submitted by qualified units in the day-ahead energy market. When bidding, these units do not have to account for the capacity that could be destined to reserves. It is the task of the TSOs/CORESO to re-dispatch these units after the closure of the day-ahead energy market and to possibly re-define their schedules in order to set the amount of capacity respectively destined to energy production and reserves. Finally, constraints (35)-(39) are the transmission limits and the cap on unserved demand are defined as in constraints (6)-(10) of Model 1, but without considering the dependence on forecast scenario $f$.

\subsubsection{TSOs/CORESO: ancillary service market}

After the closure of the day-ahead energy market, the TSOs/CORESO immediately runs the ancillary services market. When the TSOs/CORESO starts its activities, it is still D-1.

The objective function (40) is the total expected cost resulting from re-dispatching the dispatctable units and from operating the real-time balancing market. The first line of (40) represents the first stage cost (day ahead) and comprises the cost of the power re-dispatched by dispatchable units and the unserved demand cost that could be found at that stage. Parameter $p_{g t}^{\mathrm{DA} *}$ represents the power scheduled by generating unit $g$ in the day-ahead energy market, being an input data to the TSOs/CORESO's problem. The second and third lines are related to the expected cost of the balancing market, as stated for Model 1 in (1).

$$
\begin{aligned}
& \operatorname{Min} \sum_{t=1}^{\mathrm{N}_{\mathrm{T}}} \sum_{g \in\left\{G(g, n), \Omega^{\mathrm{Di}}\right\}} C_{g}^{\mathrm{G}}\left(p_{g t}^{\mathrm{TSO}}-p_{g t}^{\mathrm{DA} *}\right)+\sum_{t=1}^{\mathrm{N}_{\mathrm{T}}} \sum_{j=1}^{\mathrm{N}_{\mathrm{J}}} C^{\mathrm{US}} d_{j t}^{\mathrm{US}{ }^{\mathrm{TSO}}}+ \\
& +\sum_{t=1}^{\mathrm{N}_{\mathrm{T}}} \sum_{s=1}^{\mathrm{N}_{\mathrm{S}}} \sum_{g \in \Omega^{\mathrm{Q}}} \xi_{s}\left[C_{g t}^{\mathrm{DUR}} r_{g t s}^{\mathrm{U}}-C_{g t}^{\mathrm{DDR}} r_{g t s}^{\mathrm{D}}\right]+ \\
& +\sum_{t=1}^{\mathrm{N}_{\mathrm{T}}} \sum_{s=1}^{\mathrm{N}_{\mathrm{S}}} \sum_{j=1}^{\mathrm{N}_{\mathrm{J}}} \xi_{s}\left[C_{j}^{\mathrm{D}}\left(\Delta d_{j t s}^{\mathrm{U}}+\Delta d_{j t s}^{\mathrm{D}}\right)+C^{\mathrm{US}} d_{j t s}^{\mathrm{US} \mathrm{RT}}\right]
\end{aligned}
$$

subject to

$$
\begin{aligned}
& \sum_{g \in\{G(g, n)\}} p_{g t}^{\mathrm{TSO}}-\sum_{j \in D(j, n)}\left(D_{j t}^{\mathrm{F}}-d_{j t}^{\mathrm{US}}{ }^{\mathrm{TSO}}\right)-\sum_{l \mid O(l)=n} p_{l t}^{\mathrm{LTSO}}+\sum_{l \mid D(l)=n} p_{l t}^{\mathrm{LTSO}}=0, \quad \forall n, \forall t, \quad\left(\lambda_{n t}^{\mathrm{TSO}}\right) \\
& \sum_{l_{z} \mid D\left(l_{z}\right)=n_{z z}} p_{l t}^{\mathrm{LTSO}}-\sum_{l_{z} \mid O(l)=n_{z z}} p_{l t}^{\mathrm{LTSO}}=\sum_{l_{z} \mid D\left(l_{z}\right)=n_{z z}} p_{l t}^{\mathrm{DA}^{*}}-\sum_{l_{z} \mid O(l)=n_{z z}} p_{l t}^{\mathrm{DA}^{*}} \quad \forall z, \forall t \quad\left(\varphi_{z t}\right) \\
& p_{l t}^{\mathrm{LTSO}}=B_{l}\left(\theta_{O(l), t}^{\mathrm{TSO}}-\theta_{D(l), t}^{\mathrm{TSO}}\right), \quad \forall l, \forall t \quad\left(\omega_{l t}^{\mathrm{P}}\right) \\
& -P_{l}^{\mathrm{Lmax}} \leq p_{l t}^{\mathrm{LTSO}} \leq P_{l}^{\mathrm{Lmax}}, \quad \forall l, \forall t \quad\left(\omega_{l t}^{\min }, \omega_{l t}^{\max }\right) \\
& -\pi \leq \theta_{n t}^{\mathrm{TSO}} \leq \pi, \quad \forall n, \forall t, \quad\left(\omega_{n t}^{\pi, \mathrm{min}}, \omega_{n t}^{\pi, \max }\right) \\
& \theta_{n t}^{\mathrm{TSO}}=0, \quad n=r e f, \forall t \quad\left(\omega_{n t}^{0}\right)
\end{aligned}
$$




$$
\begin{aligned}
& 0 \leq d_{j t}^{\mathrm{US}} \leq D_{j t}^{\mathrm{F}}, \quad \forall j, \forall t \quad\left(\psi_{j t}^{\mathrm{TSO}}\right) \\
& 0 \leq p_{g t}^{\mathrm{TSO}} \leq P_{g}^{\mathrm{Gmax}}, \quad \forall g \in \Omega^{\mathrm{nQ}}, \Omega^{\mathrm{St}}, \forall t \quad\left(\mu_{g t}^{\mathrm{TSO}}\right) \\
& p_{g t}^{\mathrm{TSO}}-p_{g, t-1}^{\mathrm{TSO}} \leq \operatorname{ramp}_{g}^{\mathrm{up}}, \quad \forall g \in \Omega^{\mathrm{nQ}}, \forall t \quad\left(\mu_{g t}^{\mathrm{rU}}\right) \\
& p_{g, t-1}^{\mathrm{TSO}}-p_{g t}^{\mathrm{TSO}} \leq \operatorname{ramp}_{g}^{\mathrm{do}}, \quad \forall g \in \Omega^{\mathrm{nQ}}, \forall t \quad\left(\mu_{g t}^{\mathrm{rD}}\right) \\
& p_{g t}^{\mathrm{TSO}}+R_{g t}^{\mathrm{U}}+R_{g t}^{\mathrm{C}} \leq P_{g}^{\mathrm{Gmax}}, \quad \forall g \in \Omega^{\mathrm{Q}}, \forall t, \quad\left(\kappa_{g t}^{\mathrm{U}}\right) \\
& p_{g t}^{\mathrm{TSO}}-R_{g t}^{\mathrm{D}} \geq 0, \quad \forall g \in \Omega^{\mathrm{Q}}, \forall t, \quad\left(\kappa_{g t}^{\mathrm{D}}\right) \\
& \left(p_{g t}^{\mathrm{TSO}}+R_{g t}^{\mathrm{U}}+R_{g t}^{\mathrm{C}}\right)-\left(p_{g, t-1}^{\mathrm{TSO}}-R_{g, t-1}^{\mathrm{D}}\right) \leq \operatorname{ramp}_{g}^{\mathrm{up}}, \quad \forall g \in \Omega^{\mathrm{Q}}, \forall t, \quad\left(\kappa_{g t}^{\mathrm{rU}}\right) \\
& \left(p_{g, t-1}^{\mathrm{TSO}}+R_{g, t-1}^{\mathrm{U}}+R_{g, t-1}^{\mathrm{C}}\right)-\left(p_{g t}^{\mathrm{TSO}}-R_{g t}^{\mathrm{D}}\right) \leq \operatorname{ramp}_{g}^{\mathrm{do}}, \quad \forall g \in \Omega^{\mathrm{Q}}, \forall t \quad\left(\kappa_{g t}^{\mathrm{rD}}\right) \\
& \sum_{g \in\left\{Z G(g, z), \Omega^{\mathrm{Q}}\right\}} R_{g t}^{\mathrm{D}} \geq K^{\mathrm{D}} \sqrt{\sum_{j \in Z D(j, z)}\left(\sigma_{j t}^{\mathrm{D}}\right)^{2}+\sum_{g \in\left\{Z G(g, z), \Omega^{\mathrm{St}}\right\}}\left(\sigma_{g t}^{\mathrm{St}}\right)^{2}}, \forall z, \forall t \quad\left(\eta_{z t}^{D}\right) \\
& \sum_{g \in\left\{Z G(g, z), \Omega^{\mathrm{Q}}\right\}} R_{g t}^{\mathrm{U}} \geq K^{\mathrm{U}} \sqrt{\sum_{j \in Z D(j, z)}\left(\sigma_{j t}^{\mathrm{D}}\right)^{2}+\sum_{g \in\left\{Z G(g, z), \Omega^{\mathrm{St}}\right\}}\left(\sigma_{g t}^{\mathrm{St}}\right)^{2}}, \forall z, \forall t \quad\left(\eta_{z t}^{U}\right) \\
& \sum_{Z_{\left.G(g, z), \Omega^{\mathrm{Q}}\right\}}} R_{g t}^{\mathrm{C}} \geq \max _{g \in\left\{Z G(g, z), \Omega^{\mathrm{Di}}\right\}}\left\{P_{g}^{\mathrm{Gmax}}\right\}, \quad \forall z, \forall t \quad\left(\eta_{z t}^{C}\right) \\
& \sum_{g \in\left\{G(g, n), \Omega^{\mathrm{Q}}\right\}}\left(r_{g t s}^{\mathrm{U}}-r_{g t s}^{\mathrm{D}}\right)+\sum_{g \in\left\{G(g, n), \Omega^{\mathrm{St}}\right\}}\left(p_{g t s}^{\mathrm{G}}-p_{g t}^{\mathrm{TSO}}\right)+ \\
& -\sum_{j \in D(j, n)}\left(D_{j t s}^{\mathrm{RT}}-D_{j t}^{\mathrm{F}}+\Delta d_{j t s}^{\mathrm{U}}-\Delta d_{j t s}^{\mathrm{D}}-d_{j t s}^{\mathrm{US}}\right)+ \\
& -\sum_{l \mid O(l)=n}\left(p_{l t s}^{\mathrm{LRT}}-p_{l t}^{\mathrm{LTSO}}\right)+\sum_{l \mid D(l)=n}\left(p_{l t s}^{\mathrm{LRT}}-p_{l t}^{\mathrm{LTSO}}\right)=0, \quad \forall n, \forall t, \forall s \quad\left(\lambda_{n t s}^{\mathrm{RT}}\right)
\end{aligned}
$$

$$
\text { Constraints }(19)-(28)
$$

Constraints (41)-(57) refer to the day ahead of the TSO's market (first stage), while constraints (58)-(59) correspond to real-time operation (second stage). The TSO re-dispatches energy at nodal level, as stated through (41), but subject to the constraint on net commercial exchanges among zones that have to remain as defined in clearing of the energy market. This corresponds to condition (42). Constraints (43)-(54) define the day-ahead scheduling and are equivalent to constraints (6)-(17) of Model 1, but without considering the dependence of the day-ahead forecast $f$. Constraints (55)-(57) impose the minimum zonal reserve needs in each period of the following day. Particularly, constraints (55) and (56) define the minimum zonal downward and upward reserve needs estimated on the basis of the volatility of the forecast errors in the demand and wind/solar production predictions. Constraint (57) establishes the minimum reserve requirement to solve the possible contingency of the largest unit. Finally, the energy balance at the real-time operation is defined through (58), while the other real-time constraints are equivalent to constraints (18)-(28) of Model 1.

\subsubsection{Model 3: Energy and reserve needs are jointly scheduled}

This model represents the energy and reserve co-optimization applied in the US and it is formulated as a two-stage stochastic program, where the first stage describes the day-ahead market and the second stage the balancing market. Figure 3 depicts the decision-making process considered for this market design.

The objective function (60) is similar to that of Model 1 , but in this case there is no dependence on the day-ahead forecasts $f$. The minimization of the objective function is subject to day-ahead (61)-(76) and real-time (77)-(78) constraints. Since the constraints of this model are equivalent to those described in the previous models, we avoid to include a detailed description for the sake of consciousness. 


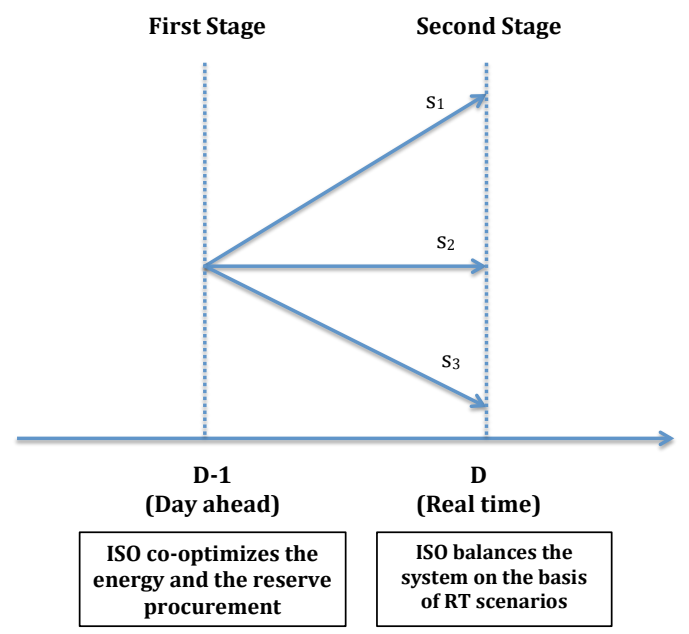

Figure 3: Decision-making process in Model 3.

$$
\begin{aligned}
& \operatorname{Min} \sum_{t=1}^{\mathrm{N}_{\mathrm{T}}}\left(\sum_{g \in\left\{G(g, n), \Omega^{\mathrm{Di}}\right\}} C_{g}^{\mathrm{G}} p_{g t}^{\mathrm{DA}}+\sum_{j=1}^{\mathrm{N}_{\mathrm{J}}} C^{\mathrm{US}} d_{j t}^{\mathrm{US}{ }^{\mathrm{DA}}}\right) \\
& +\sum_{t=1}^{\mathrm{N}_{\mathrm{T}}} \sum_{s=1}^{\mathrm{N}_{\mathrm{S}}} \sum_{g \in \Omega^{\mathrm{Q}}} \xi_{s}\left[C_{g t}^{\mathrm{DUR}} r_{g t s}^{\mathrm{U}}-C_{g t}^{\mathrm{DDR}} r_{g t s}^{\mathrm{D}}\right]+ \\
& +\sum_{t=1}^{\mathrm{N}_{\mathrm{T}}} \sum_{s=1}^{\mathrm{N}_{\mathrm{S}}} \sum_{j=1}^{\mathrm{N}_{\mathrm{J}}} \xi_{s}\left[C_{j}^{\mathrm{D}}\left(\Delta d_{j t s}^{\mathrm{U}}+\Delta d_{j t s}^{\mathrm{D}}\right)+C^{\mathrm{US}} d_{j t s}^{\mathrm{US}}\right]
\end{aligned}
$$

subject to

$$
\begin{aligned}
& \sum_{g \in G(g, n)} p_{g t}^{\mathrm{DA}}-\sum_{j \in D(j, n)}\left(D_{j t}^{\mathrm{F}}-d_{j t}^{\mathrm{US}}{ }^{\mathrm{DA}}\right)-\sum_{l \mid O(l)=n} p_{l t}^{\mathrm{LDA}}+\sum_{l \mid D(l)=n} p_{l t}^{\mathrm{LDA}}=0, \quad \forall n, \forall t \quad\left(\lambda_{n t}^{\mathrm{DA}}\right) \\
& p_{l t}^{\mathrm{LDA}}=B_{l}\left(\theta_{O(l), t}^{\mathrm{DA}}-\theta_{D(l), t}^{\mathrm{DA}}\right), \quad \forall l, \forall t \quad\left(\rho_{l t}^{\mathrm{p}}\right) \\
& -P_{l}^{\mathrm{Lmax}} \leq p_{l t}^{\mathrm{LDA}} \leq P_{l}^{\mathrm{Lmax}}, \quad \forall l, \forall t \quad\left(\rho_{l t}^{\min }, \rho_{l t}^{\max }\right) \\
& -\pi \leq \theta_{n t}^{\mathrm{DA}} \leq \pi, \quad \forall n, \forall t \quad\left(\rho_{n t}^{\pi, \min }, \rho_{n t}^{\pi, \max }\right) \\
& \quad \theta_{n t}^{\mathrm{DA}}=0, \quad n=r e f, \forall t \quad\left(\rho_{n t}^{0}\right) \\
& 0 \leq d_{j t}^{\mathrm{US}} \leq D_{j t}^{\mathrm{DA}}, \quad \forall j, \forall t \quad\left(\psi_{j t}^{\mathrm{DA}}\right) \\
& 0 \leq p_{g t}^{\mathrm{DA}} \leq P_{g}^{\mathrm{Gmax}}, \quad \forall g \in \Omega^{\mathrm{nQ}}, \Omega^{\mathrm{St}}, \forall t \quad\left(\mu_{g t}\right) \\
& p_{g t}^{\mathrm{DA}}-p_{g, t-1}^{\mathrm{DA}} \leq \operatorname{ramp} p_{g}^{\mathrm{up}}, \quad \forall g \in \Omega^{\mathrm{nQ}}, \forall t \quad\left(\mu_{g t}^{r U}\right) \\
& p_{g, t-1}^{\mathrm{DA}}-p_{g t}^{\mathrm{DA}} \leq \operatorname{ramp}_{g}^{\mathrm{do}}, \quad \forall g \in \Omega^{\mathrm{nQ}}, \forall t \quad\left(\mu_{g t}^{r D}\right) \\
& p_{g t}^{\mathrm{DA}}+R_{g t}^{\mathrm{U}}+R_{g t}^{\mathrm{C}} \leq P_{g}^{\mathrm{Gmax}}, \quad \forall g \in \Omega^{\mathrm{Q}}, \forall t \quad\left(\kappa_{g t}^{\mathrm{U}}\right) \\
& p_{g t}^{\mathrm{DA}}-R_{g t}^{\mathrm{D}} \geq 0, \quad \forall g \in \Omega^{\mathrm{Q}}, \forall t \quad\left(\kappa_{g t}^{\mathrm{D}}\right) \\
& \left(p_{g t}^{\mathrm{DA}}+R_{g t}^{\mathrm{U}}+R_{g t}^{\mathrm{C}}\right)-\left(p_{g, t-1}^{\mathrm{DA}}-R_{g, t-1}^{\mathrm{D}}\right) \leq \operatorname{ramp}_{g}^{\mathrm{up}}, \quad \forall g \in \Omega^{\mathrm{Q}}, \forall t \quad\left(\kappa_{g t}^{r U}\right) \\
& \left(p_{g, t-1}^{\mathrm{DA}}+R_{g, t-1}^{\mathrm{U}}+R_{g, t-1}^{\mathrm{C}}\right)-\left(p_{g t}^{\mathrm{DA}}-R_{g t}^{\mathrm{D}}\right) \leq \operatorname{ramp}_{g}^{\mathrm{do}}, \quad \forall g \in \Omega^{\mathrm{Q}}, \forall t \quad\left(\kappa_{g t}^{r D}\right)
\end{aligned}
$$




$$
\begin{aligned}
& \sum_{g \in\left\{Z G(g, z), \Omega^{\mathrm{Q}}\right\}} R_{g t}^{\mathrm{D}} \geq K^{\mathrm{D}} \sqrt{\sum_{j \in Z D(j, z)}\left(\sigma_{j t}^{\mathrm{D}}\right)^{2}+\sum_{g \in\left\{Z G(g, z), \Omega^{\mathrm{St}}\right\}}\left(\sigma_{g t}^{\mathrm{St}}\right)^{2}}, \quad \forall z, \forall t \quad\left(\eta_{z t}^{D}\right) \\
& \sum_{g \in\left\{Z G(g, z), \Omega^{\mathrm{Q}}\right\}} R_{g t}^{\mathrm{U}} \geq K^{\mathrm{U}} \sqrt{\sum_{j \in Z D(j, z)}\left(\sigma_{j t}^{\mathrm{D}}\right)^{2}+\sum_{g \in\left\{Z G(g, z), \Omega^{\mathrm{St}}\right\}}\left(\sigma_{g t}^{\mathrm{St}}\right)^{2}}, \forall z, \forall t \quad\left(\eta_{z t}^{U}\right) \\
& \quad \sum_{g \in\left\{Z G(g, z), \Omega^{\mathrm{Q}}\right\}} R_{g t}^{\mathrm{C}} \geq \max _{g \in\left\{Z G(g, z), \Omega^{\mathrm{Di}}\right\}}\left\{P_{g}^{\mathrm{Gmax}}\right\}, \quad \forall z, \forall t \quad\left(\eta_{z t}^{C}\right) \\
& \sum_{g \in\left\{G(g, n), \Omega^{\mathrm{Q}}\right\}}\left(r_{g t s}^{\mathrm{U}}-r_{g t s}^{\mathrm{D}}\right)+\sum_{g \in\left\{G(g, n), \Omega^{\mathrm{St}}\right\}}\left(p_{g t s}^{\mathrm{G}}-p_{g t}^{\mathrm{DA}}\right)+ \\
& -\sum_{j \in D(j, n)}\left(D_{j t s}^{\mathrm{RT}}-D_{j t}^{\mathrm{F}}+\Delta d_{j t s}^{\mathrm{U}}-\Delta d_{j t s}^{\mathrm{D}}-d_{j t s}^{\mathrm{US}}\right)+ \\
& -\sum_{l \mid O(l)=n}\left(p_{l t s}^{\mathrm{LRT}}-p_{l t}^{\mathrm{LDA}}\right)+\sum_{l \mid D(l)=n}\left(p_{l t s}^{\mathrm{LRT}}-p_{l t}^{\mathrm{LDA}}\right)=0, \quad \forall n, \forall t, \forall s \quad\left(\lambda_{n t s}^{\mathrm{RT}}\right) \\
& \text { Constraints }(19)-(28)
\end{aligned}
$$

\section{Case Study: input data and assumptions}

This section provides a brief description of the input data used in the case study and additional assumptions on which our case study is based on. Due to space limitations, the tables reporting the input data related to the generating units and the transmission system are described in $\mathrm{C}$.

The numerical analysis is conducted on the IEEE 24-node Reliability Test System (RTS). ${ }^{17}$ We consider an updated version of the generating system of the RTS, where the renewable capacity represents $47.5 \%$ of the total generating capacity in the system.

We generate day-ahead and real-time scenarios of the uncertain parameters (wind and solar power production and demand level) as described in D. Specifically, we consider 3 scenarios for the day ahead from which we generate 10 real-time scenarios giving a total of 30 real-time scenarios. Due to the structure of the market, Model 1 has been run considering the 3 day-ahead forecast scenarios and the 30 real-time scenarios all together. However, Model 2 and Model 3 are run three times, one per each day-ahead forecast and considering the corresponding 10 real-time scenarios of each day-ahead forecast.

As explained in Section 2.1, reserves are procured at zonal level and the minimum capacity requirements depend on the degree of coordination of the TSOs. The characteristics of the two cases studied in this paper are the following ones:

1) Three zones case: This envisages the "not-coordinated reserve procurement" where there is no coordination among the TSOs and hence, reserve needs are independently defined by each TSO in its zone (zonal representation). We consider the three-zone subdivision in the network as represented in Figure C.7 in C. This way, the conventional reserve requirement for zones 1,2 and 3 is $300 \mathrm{MW}, 400 \mathrm{MW}$ and $500 \mathrm{MW}$, respectively, which corresponds to the capacity of the largest unit located in each zone. Therefore, the total capacity devoted to conventional reserves has to be, at least, equal to $1200 \mathrm{MW}$. Note that in Model 2 the constraint on cross-zonal day-ahead energy flow limitations (42) is enforced. However, as explained in Section 1.3, we assume perfectly coordinated balancing markets in all models, and hence, cross-border constraints are not considered in the real-time market of Model 2.

2) One zone case: This represents the "coordinated reserve procurement", which can be interpreted as a situation where a single TSO procures reserves for the whole system. The three models are directly adapted by setting index $z$ equal to 1 . In addition, in Model 2 the coordinate reserve procurement implies that the TSO can operate the ancillary services market without the requirement of maintaining cross-border day-ahead energy flows, i.e., constraint (42) does not apply in this case. Finally, the conventional reserve requirement in this case is $500 \mathrm{MW}$, which corresponds to the capacity of the largest unit of the whole system.

All cases have been solved using CPLEX 12.6.3.0 (http://www.ilog.com/products/cplex/) under GAMS 24.6.1 on a Intel(R) Core(TM) $1.30 \mathrm{GHz}$ processor with 4 GB RAM. Finally, Table 2 reports the computation time of three models run under the different assumptions considered.

\footnotetext{
${ }^{17}$ Reliability Test System Task Force (1999). The IEEE reliability test system-1996, IEEE Transaction on Power Systems, 14(3), 1010-1020.
} 


\begin{tabular}{|c|c|c|c|c|c|}
\hline & & & ee zon & & \\
\hline & Model 1 & Mod & & Mod & \\
\hline \multirow{3}{*}{ Computation time } & \multirow{3}{*}{$28.64 \mathrm{~s}$} & Forecast 1 & $2.13 \mathrm{~s}$ & Forecast 1 & $2.19 \mathrm{~s}$ \\
\hline & & Forecast 2 & $1.75 \mathrm{~s}$ & Forecast 2 & $1.19 \mathrm{~s}$ \\
\hline & & Forecast 3 & $1.77 \mathrm{~s}$ & Forecast 3 & $1.83 \mathrm{~s}$ \\
\hline & \multicolumn{5}{|c|}{ One zone } \\
\hline & Model 1 & \multicolumn{2}{|c|}{ Model 2} & \multicolumn{2}{|c|}{ Model 3} \\
\hline \multirow{3}{*}{ Computation time } & \multirow{3}{*}{$15.15 \mathrm{~s}$} & Forecast 1 & $1.33 \mathrm{~s}$ & Forecast 1 & $1.31 \mathrm{~s}$ \\
\hline & & Forecast 2 & $1.43 \mathrm{~s}$ & Forecast 2 & $1.44 \mathrm{~s}$ \\
\hline & & Forecast 3 & $1.36 \mathrm{~s}$ & Forecast 3 & $1.36 \mathrm{~s}$ \\
\hline
\end{tabular}

Table 2: Computation time of the different models (measured in seconds)

\section{General Discussion}

The number of considered scenarios may be small and hence our results may look at best illustrative, but they reflect basic economic phenomena. In particular, Model 3 (co-optimization of energy and reserves) is expected to be more efficient than Models 1 and 2. Model 2 (reserves after the energy market) partially reproduces the co-optimized procurement of energy and reserves in the ancillary services market. The TSO can correct the schedule of the day-ahead energy market if necessary by partially discarding its result and conducting an ex-post co-optimization of energy and reserve. However, the interest of Model 2 is to assess the impact of imposing the cross-border exchange scheduled in the day-ahead energy market to the ancillary services market in the zonal representation. Thus, while in the one zone case Model 2 represents a good proxy of the co-optimization paradigm proposed in Model 3, in the three zones case the imposition of cross-zonal constraints leads to welfare losses. Finally, Model 1 (reserves before the energy market) is expected to be the farthest from Model 3 in terms of system costs.

These principles are general even though not always recognized in practice. They are confirmed by the numerical results that we discuss in the following sections. However, a dedicated analysis would be necessary to obtain a precise numerical evaluation for the European electricity market.

Furthermore, this analysis suggests some side questions. Flexility is an often mentioned subject in the context of renewable energy sources. A relevant question is whether one should introduce a special flexibility market for dealing with variable renewable sources (which would require defining flexibility services) or whether the flexibility of the existing plants of the system is sufficient (in which case a market for flexibility would be redundant). This suggests analyzing results in terms of the following stylized questions:

1. Could it be that the flexibility embedded in plant capacity is sufficient? This would mean that flexibility is effectively a byproduct of existing capacity and there is no need for a flexibility market. We refer to that situation as to a capacity driven market.

2. In contrast the flexibility offered by existing plants could be tight and fully used. This could constraint and hence modify the merit order, making it necessary to introduce a market for ramping services. We refer to that situation as to a capacity and flexibility driven market.

In this case study, the analysis of the problem is conduced by comparing the autarky and integrated markets. The results provided in Subsection 5.3 show that from the capacity driven paradigm observed in the coordinated case, where capacity offers flexibility as a byproduct, we move towards a flexibility tight market in the notcoordinated case, where flexibility is no longer a byproduct of capacity but it is remunerated on its own. This is particularly evident for CCGT units, which are mainly used for ramping.

\section{Results}

This section reports the numerical results obtained from the models under study. Specifically, we compare the following items for the three zones and one zone cases: $i)$ the scheduled reserve capacities and the renewable power spillage; ii) the system operating costs; iii) the generating units' profits; and iv) the consumers' benefits and the TSOs/CORESO's remuneration.

\subsection{Scheduled reserves and renewable power spillage}

To allow a better understanding of the economic results obtained from each market structure, we firstly show how reserves are scheduled and the renewable power spillage in each model. Figure 4 depicts the use of the CCGT units to provide conventional and upward reserve capacity and energy in the day-ahead market. The capacity that is not used is also indicated. Comparing the not-coordinated and the coordinated cases, we observe that the three-zone system requires that the CCGT units provide a comparatively higher conventional reserve 
capacity, which results in lower flexibility in the system operation. The more significant differences in the reserve capacity scheduled in each model are found when the power system is in a comparatively flexible situation. In the three-zones case, a comparable more flexible situation corresponds to the first and last hours of the day. Model 1 schedules higher reserve capacity than the other two models. Instead, in the one-zone case, this is more evident in general because there are no zonal limitations to procure the reserve needs so it makes the system quite flexible. Again, we observe that higher reserve capacity is scheduled in Model 1 with respect to Model 2 and Model 3.
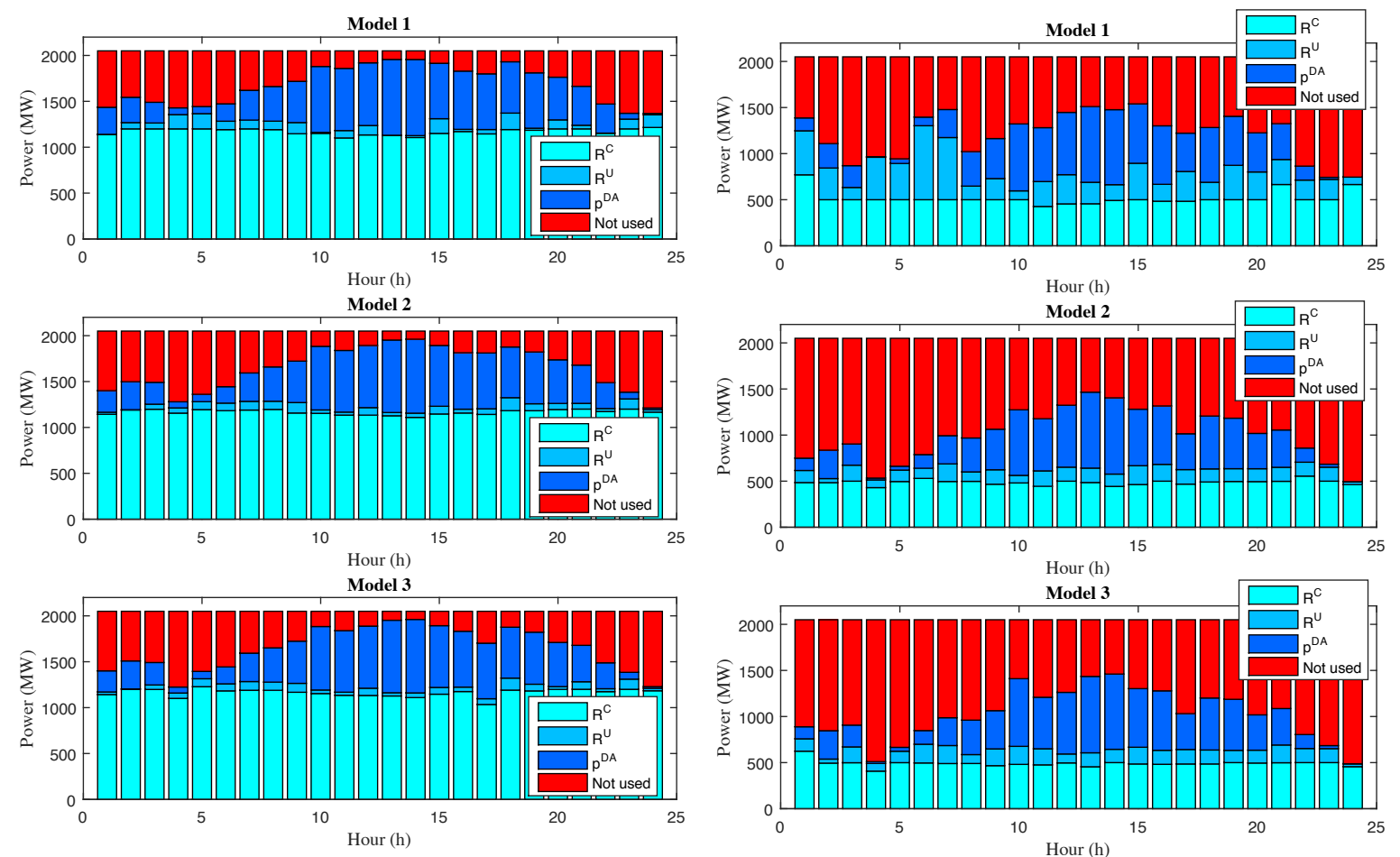

(a) Three zones

(b) One zone

Figure 4: Use of CCGT units in the day ahead.

Figure 5 shows the wind and solar power spillage in each model and for the not-coordinated and coordinated cases. As it can be observed, the lack of coordination in the reserve procurement entails a less efficient use of the renewable power. The reason is that the higher use of CCGT units in providing reserve capacity makes the system less flexible and hence, it becomes more difficult to counteract the variability of the renewable sources. 

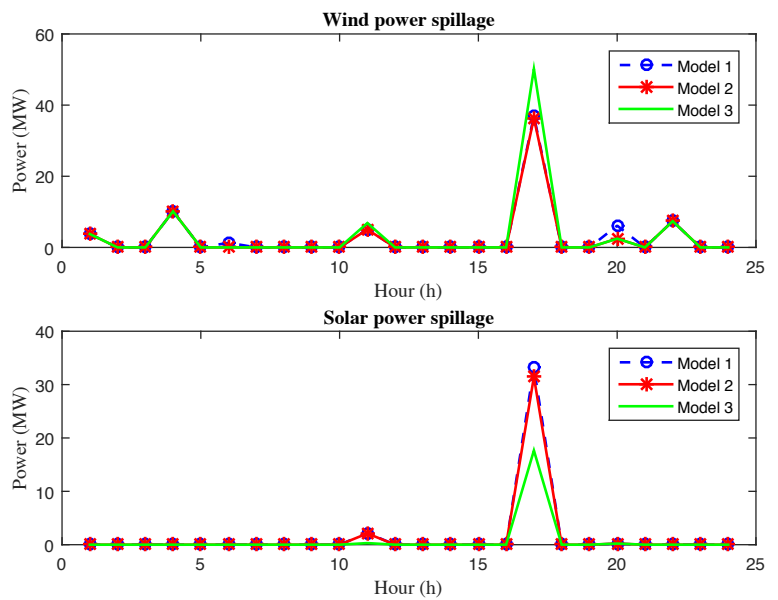

(a) Three zones

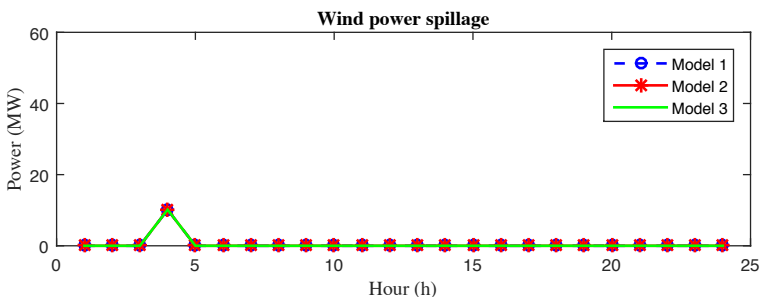

Solar power spillage

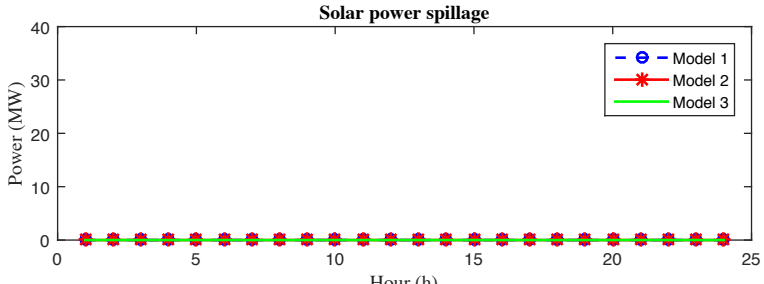

(b) One zone

Figure 5: Wind and solar power spillage in each model and case.

\subsection{System's operating costs}

The system daily operating costs in the three models for the two cases are given in Tables 3-5. Costs are subdivided into "DA energy costs", "RT operating costs", and "RT unserved demand value" that represent the costs associated with the day-ahead energy scheduling, the real-time operating costs, and the penalization of the involuntary unserved demand, respectively. The real time costs are related to upward/downward reserve utilization and the DSM services provided by consumers. Tables 4 and 5 report the results obtained for each forecast and the relative expectation. More specifically, in Table 4, we first illustrate the outcomes of the PXs/PCR and of the TSOs/CORESO problems, separately, and, then, we put them together to make them comparable with the results of the other two models.

\begin{tabular}{l|c|c}
\hline & Three zones & One zone \\
\hline Total operating cost & $\mathbf{1 , 3 8 8 , 9 2 5}$ & $\mathbf{1 , 0 9 7 , 4 1 6}$ \\
\hline DA energy cost & $1,124,847$ & $1,092,812$ \\
RT operating cost & 24,726 & 4,604 \\
RT unserved demand value & 239,230 & 0 \\
\hline
\end{tabular}

Table 3: Total system costs (\$) in Model 1 (daily values)

\begin{tabular}{|c|c|c|c|c|c|}
\hline & & \multicolumn{3}{|c|}{ Three zones } & \multirow[b]{2}{*}{ Expected } \\
\hline & & Forecast 1 & Forecast 2 & Forecast 3 & \\
\hline PXs & DA energy costs & $1,062,997$ & 950,675 & $1,231,596$ & $1,081,756$ \\
\hline \multirow{4}{*}{ TSOs } & Total TSOs costs & 357,304 & 117,577 & 417,898 & 297,693 \\
\hline & DA re-dispatch costs & 24,047 & 45,829 & 16,836 & 28,904 \\
\hline & RT operating costs & 29,601 & 24,239 & 34,834 & 29,558 \\
\hline & RT unserved demand value & 303,655 & 47,808 & 366,227 & 239,230 \\
\hline \multirow{4}{*}{ PXs+TSOs } & Total operating costs & $1,420,301$ & $1,068,553$ & $1,649,493$ & $1,379,349$ \\
\hline & DA energy costs & $1,087,044$ & 996,505 & $1,248,432$ & $1,110,660$ \\
\hline & RT operating costs & 29,601 & 24,239 & 34,834 & 29,558 \\
\hline & RT unserved demand value & 303,655 & 47,808 & 366,227 & 239,230 \\
\hline & & \multicolumn{3}{|c|}{ One zone } & \\
\hline & & Forecast 1 & Forecast 2 & Forecast 3 & Expected \\
\hline PXs & DA energy costs & $1,062,997$ & 950,675 & $1,231,596$ & $1,081,756$ \\
\hline \multirow{4}{*}{ TSOs } & Total TSOs costs & 22,070 & 15,188 & 3,879 & 13,712 \\
\hline & DA re-dispatch costs & 7,173 & 3,988 & $-5,538$ & 1,874 \\
\hline & RT operating costs & 14,897 & 11,200 & 9,416 & 11,838 \\
\hline & RT unserved demand value & 0 & 0 & 0 & 0 \\
\hline \multirow{4}{*}{ PXs+TSOs } & Total operating costs & $1,085,067$ & 965,864 & $1,235,474$ & $1,095,468$ \\
\hline & DA energy costs & $1,070,170$ & 954,664 & $1,226,058$ & $1,083,630$ \\
\hline & RT operating costs & 14,897 & 11,200 & 9,416 & 11,838 \\
\hline & RT unserved demand value & 0 & 0 & 0 & 0 \\
\hline
\end{tabular}

Table 4: Total system costs (\$) in Model 2 (daily values) 


\begin{tabular}{l|ccc|c}
\hline & \multicolumn{3}{|c|}{ Three zones } & \\
& Forecast 1 & Forecast 2 & Forecast 3 & Expected \\
\hline Total operating costs & $1,420,301$ & $1,068,253$ & $1,649,007$ & $\mathbf{1 , 3 7 9 , 1 8 7}$ \\
\hline DA energy costs & $1,085,844$ & 995,057 & $1,247,155$ & $1,109,352$ \\
RT operating costs & 30,801 & 25,387 & 35,625 & 30,604 \\
RT unserved demand value & 303,656 & 47,809 & 366,227 & 239,231 \\
\hline \hline & & One zone & \\
& Forecast 1 & Forecast 2 & Forecast 3 & Expected \\
\hline Total operating costs & $1,085,067$ & 965,864 & $1,235,474$ & $\mathbf{1 , 0 9 5 , 4 6 8}$ \\
\hline DA energy costs & $1,073,443$ & 949,631 & $1,231,071$ & $1,084,715$ \\
RT operating costs & 11,624 & 16,233 & 4,403 & 10,753 \\
RT unserved demand value & 0 & 0 & 0 & 0 \\
\hline
\end{tabular}

Table 5: Total system costs (\$) in Model 3 (daily values)

The main results of this analysis are as follows. First, the most relevant consequence of the no coordination in the reserve procurement is the involuntary unserved demand found in the real-time operation (compare, for instance, the values in the last line of Table 3 between the three zones and one zone cases). The expected unserved demand in the three zones case is $24 \mathrm{MWh}$ for all models, which occurs in some scenarios due to the reduction in the available generating capacity as a consequence of the reserve capacity requirements. The segmentation of the power system into three reliability zones implies a higher requirement of conventional reserves compared to the coordinated case, going from $500 \mathrm{MW}$ to $1200 \mathrm{MW}$. These results show the negative effect of operating a power system with high renewable capacity and no coordination in the procurement of reserve needs. Indeed, the system operation in reality is much more flexible and secure than what our models simulate, but these results give a quantitative idea of those effects.

Second, the positive value of the real-time operating costs indicates that, in general, the upward reserve is more frequently deployed than the downward reserve. As we assume that stochastic units can be scheduled up to their capacity in the day-ahead market, it is less costly for the system to schedule stochastic units in an optimistic way in the day-ahead energy market and then to deploy upward reserve in the real-time operation when needed.

Third, procuring reserves before the clearing of the day-ahead energy market (Model 1) turns out to be the least efficient option in both the not-coordinated and coordinated cases. Since the reserve schedule is based on less information (a distribution of forecast errors instead of a day-ahead forecast), the TSO procures more reserves than effectively needed by the system. This implies higher total operating costs.

Fourth, Model 2 is not as efficient as Model 3 in the three-zones case. The imposition of the cross-border constraints in the day ahead of the ancillary services market of Model 2 limits the action of the TSO, which leads to slightly higher operating costs in Model 2 compared to Model 3. However, looking at the results corresponding to the one zone case, Model 2 is as efficient as Model 3.

Finally, note that Tables 3-5 report daily values. If this reasoning is translated in annual terms, assuming a similar variability of the daily scheduling along the year, the impact that the inefficiency of Model 1 has in terms of its extra-costs (money loss) compared to Model 2 and Model 3 may be significant, as shown in Table 6.

\begin{tabular}{l|cc|cc}
\hline & \multicolumn{2}{|c|}{ Three zones } & \multicolumn{2}{c}{ One zone } \\
\cline { 2 - 5 } & Daily & Annually & Daily & Annually \\
\hline Model 1 vs. Model 2 & 9,576 & $3,495,240$ & 1,947 & 710,655 \\
Model 1 vs. Model 3 & 9,738 & $3,554,370$ & 1,947 & 710,655 \\
Model 2 vs. Model 3 & 162 & 59,130 & 0 & 0 \\
\hline
\end{tabular}

Table 6: Extra operating costs $(\$)$ among models

\subsection{Generating units' profits}

The generators' profits obtained by each technology in each model in the three-zones and one-zone cases are reported in Tables 7 and 8, respectively. As explained in B, the profits of the generators come from two sources: i) the energy and reserve services, and $i$ i) the flexibility services, linked to their ramping capability.

In the three zone case, the units' profits are so high because of the effect of the unserved demand registered in the real-time operation. This effect distorts the results and, for this reason, we analyze in more detail the unit profits in the coordinated case, where no unserved demand is found. 


\begin{tabular}{|c|c|c|c|c|c|}
\hline & \multicolumn{5}{|c|}{ Three Zones } \\
\hline & & & Model 1 & $\begin{array}{c}\text { Model 2 } \\
\text { (Expected) }\end{array}$ & $\begin{array}{c}\text { Model 3 } \\
\text { (Expected) }\end{array}$ \\
\hline \multirow{8}{*}{ Qualified units } & \multirow{4}{*}{ Coal } & Energy and Reserves & $2,173,000$ & $2,182,569$ & $2,183,230$ \\
\hline & & Upward ramping & 9,389 & 6,747 & 6,658 \\
\hline & & Downward ramping & 3,891 & 5,065 & 5,154 \\
\hline & & Total profit & $2,186,280$ & $2,194,382$ & $2,195,043$ \\
\hline & \multirow{4}{*}{ CCGT } & Energy and Reserves & $5,468,159$ & $5,515,309$ & $5,515,896$ \\
\hline & & Upward ramping & $1,116,705$ & $1,092,630$ & $1,091,753$ \\
\hline & & Downward ramping & 165,612 & 146,707 & 147,400 \\
\hline & & Total profit & $6,750,476$ & $6,754,646$ & $6,755,049$ \\
\hline \multirow{4}{*}{ Not-qualified units } & \multirow{4}{*}{ Nuclear } & Energy & $2,142,605$ & $2,151,942$ & $2,151,942$ \\
\hline & & Upward ramping & 100 & 100 & 100 \\
\hline & & Downward ramping & - & - & - \\
\hline & & Total profit & $2,142,705$ & $2,152,042$ & $2,152,042$ \\
\hline \multirow{6}{*}{ Stochastic units } & \multirow{3}{*}{ Wind } & Energy & $2,312,859$ & $2,336,461$ & $2,336,448$ \\
\hline & & Spillage in RT & - & - & - \\
\hline & & Total profit & $2,312,859$ & $2,336,461$ & $2,336,448$ \\
\hline & \multirow{3}{*}{ Solar } & Energy & $1,370,510$ & $1,375,993$ & $1,375,991$ \\
\hline & & Spillage in RT & - & - & - \\
\hline & & Total profit & $1,370,510$ & $1,375,993$ & $1,375,991$ \\
\hline
\end{tabular}

Table 7: Generating units' profits $(\$)$ in the three models in the three zones case (daily values)

\begin{tabular}{|c|c|c|c|c|c|}
\hline & \multicolumn{5}{|c|}{ One Zone } \\
\hline & & & Model 1 & $\begin{array}{c}\text { Model 2 } \\
\text { (Expected) }\end{array}$ & $\begin{array}{c}\text { Model 3 } \\
\text { (Expected) }\end{array}$ \\
\hline \multirow{8}{*}{ Qualified units } & \multirow{4}{*}{ Coal } & Energy and Reserves & 115,459 & 115,688 & 115,688 \\
\hline & & Upward ramping & 121 & 7 & 13 \\
\hline & & Downward ramping & 1,460 & 1,548 & 1,542 \\
\hline & & Total profit & 117,040 & 117,243 & 117,243 \\
\hline & \multirow{4}{*}{ CCGT } & Energy and Reserves & 9,434 & 9,361 & 9,361 \\
\hline & & Upward ramping & 3,613 & 3,277 & 3,453 \\
\hline & & Downward ramping & 2,278 & 2,400 & 2,224 \\
\hline & & Total profit & 15,326 & 15,038 & 15,038 \\
\hline \multirow{4}{*}{ Not-qualified units } & \multirow{4}{*}{ Nuclear } & Energy & 440,185 & 441,440 & 441,440 \\
\hline & & Upward ramping & - & - & - \\
\hline & & Downward ramping & - & - & - \\
\hline & & Total profit & 440,185 & 441,440 & 441,440 \\
\hline \multirow{3}{*}{ Stochastic units } & \multirow[t]{2}{*}{ Wind } & $\begin{array}{l}\text { Energy } \\
\text { Spillage in RT }\end{array}$ & 818,493 & 822,260 & 822,260 \\
\hline & & Total profit & 818,493 & 822,260 & 822,260 \\
\hline & Solar & $\begin{array}{l}\text { Energy } \\
\text { Spillage in RT } \\
\text { Total profit }\end{array}$ & $\begin{array}{c}241,926 \\
- \\
\mathbf{2 4 1 , 9 2 6}\end{array}$ & $\begin{array}{c}242,205 \\
- \\
\mathbf{2 4 2 , 2 0 5}\end{array}$ & $\begin{array}{c}242,205 \\
- \\
\mathbf{2 4 2 , 2 0 5}\end{array}$ \\
\hline
\end{tabular}

Table 8: Generating units' profits (\$) in the three models in the one zone case

As it can be seen in Table 8, the best economic outcomes obtained by the generators result from Model 2 and Model 3, except for the CCGT units. These units provide more reserve capacity in Model 1 and, hence, they obtain a higher income. Wind units get the best economic outcomes independently of the market organization.

Looking in more details at the qualified units, we observe that coal units, with lower operating costs than CCGT plants, supply high share of the energy, from where they obtain most of their profits. The remaining part of their capacity is mainly employed for reserves. Since coal units are online during most of the day, they provide high downward reserve capacity, as it can be noticed from their downward ramping profits. On the other hand, the profits obtained by CCGT units are much smaller than the rest of the generators due to their high operating costs and low participation to the day-ahead energy market. Their profits mainly derive from reserves commitment and ramping services that are employed to guarantee flexibility to the system (note that the amount of reserves scheduled with CCGT plants is higher than that procured by coal units).

Comparing the results by looking at the level of coordination in the reserve procurement, we observe that while capacity, as a provider of energy and reserve, is the main source of revenue in the coordinated case, the role of flexibility becomes more important when this coordination disappears (compare Tables 7 and 8).

Figure 6 depicts the expected day-ahead prices resulting from each model in the three-zones and one-zone cases. The graphs show that the electricity prices in the day-ahead energy market are slightly more stable in Model 2 than in Models 1 and 3, specially in the three zones case. In this case, the day-ahead energy prices in Model 1 and Model 3 adopt a very high value in those hours in which there is unserved demand in the real-time operation, even though there is no unserved demand in the day-ahead market. Assuming that power system operations are much more flexible as what it is represented in the proposed models, the fact that the day-ahead energy market, where most of the energy is dispatched, does not give significant differences among the hourly prices may be a good feature of the market represented by Model 2. 


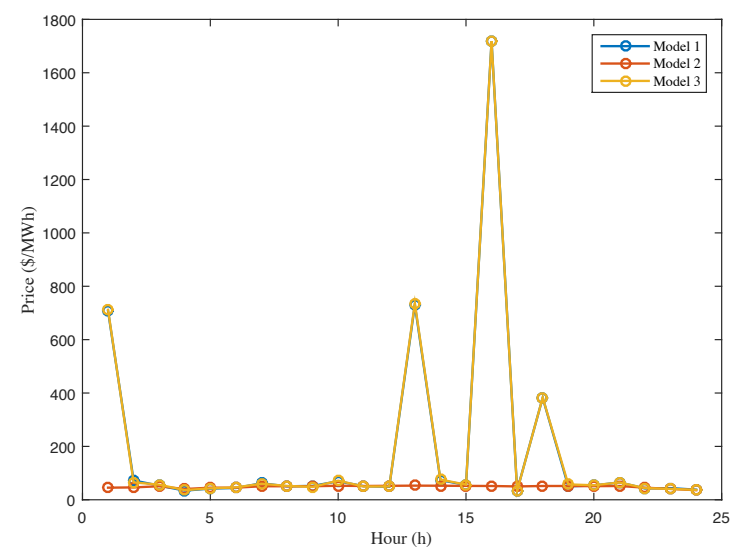

(a) Three zones

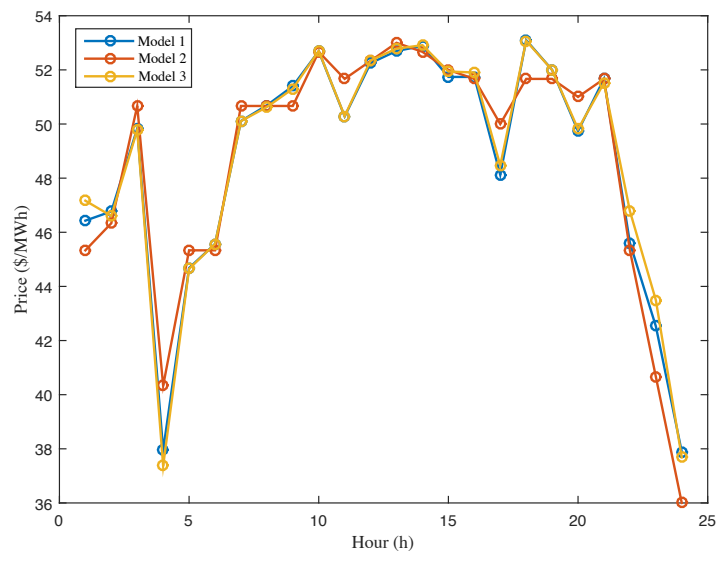

(b) One zone

Figure 6: Day-ahead energy prices in each model and case.

\subsection{Consumers' benefit and TSO's remuneration}

Table 9 reports the consumers' benefit, which accounts for the values of energy services and the reliability provided by the scheduled reserves. Considering the energy services, we report the consumers' willingness to pay ("Willingness to pay"), their revenues from DSM services ("Remuneration of DSM services in RT"), the costs they face for buying electricity in the day-ahead energy market ("Electricity payment in DA"), and the value of the deviation between the actual demand in real time and the forecast in day ahead ("Value of demand deviation"). In addition, for Model 2 only, we indicate the re-dispatching costs, coming from the re-scheduling of the dispatchable units that the TSOs/CORESO imposes on consumers (see "Re-dispatch costs" in Table 9). In all models, the "Value of demand deviation" represents a cost that is charged to consumers.

Generally, consumers' benefit is lower when there is no coordination in reserve procurement. In particular, in the three zones case, the lowest consumers' benefit value is registered in Model 3. Since Model 3 represents the most efficient market organization among the ones analyzed, the higher benefits obtained in Models 1 and 2 must be due to some system inefficiencies. However, in Model 2 the sum of the terms "Electricity payment in $D A$ " and "Re-dispatch costs" results to be equal to $\$ 12,399,907$, which is very close to the "electricity payment in day ahead" of Model 3 (it is exactly the same in the coordinated case). Moreover, the cost faced by consumers for the system reliability in Model 1 is higher than in the other two market paradigms, which is linked with the reserve procurement.

\begin{tabular}{|c|c|c|c|c|}
\hline & & \multicolumn{3}{|c|}{ Three zones } \\
\hline & & Model 1 & $\begin{array}{c}\text { Model 2 } \\
\text { (Expected) }\end{array}$ & $\begin{array}{c}\text { Model 3 } \\
\text { (Expected) }\end{array}$ \\
\hline \multirow{5}{*}{ Energy services } & Willingness to pay & $539,122,936$ & $539,122,936$ & $539,122,936$ \\
\hline & Remuneration of DSM services in RT & 471,842 & 469,464 & 469,456 \\
\hline & Electricity payment in DA & $12,353,266$ & $2,780,955$ & $12,401,545$ \\
\hline & Value of demand deviation & 37,441 & 36,759 & 36,772 \\
\hline & Re-dispatch costs & n.a. & $9,618,952$ & n.a. \\
\hline \multirow[t]{4}{*}{ Reliability } & Value of the system reliability & $4,274,302$ & $4,266,867$ & $4,266,985$ \\
\hline & Consumers' benefit & $522,929,769$ & $522,888,867$ & $522,887,090$ \\
\hline & & \multicolumn{3}{|c|}{ One zone } \\
\hline & & Model 1 & $\begin{array}{c}\text { Model 2 } \\
\text { (Expected) }\end{array}$ & $\begin{array}{c}\text { Model 3 } \\
\text { (Expected) }\end{array}$ \\
\hline \multirow{5}{*}{ Energy services } & Willingness to pay & $539,122,936$ & $539,122,936$ & $539,122,936$ \\
\hline & Remuneration of DSM services in RT & 8 & 5 & 5 \\
\hline & Electricity payment in DA & $2,780,407$ & $2,780,955$ & $2,784,559$ \\
\hline & Value of demand deviation & 962 & 943 & 943 \\
\hline & Re-dispatch costs & n.a. & 3,604 & n.a. \\
\hline \multirow[t]{2}{*}{ Reliability } & Value of the system reliability & 583 & 602 & 602 \\
\hline & Consumers' benefit & $536,340,992$ & $536,336,837$ & $536,336,837$ \\
\hline
\end{tabular}

Table 9: Consumers' benefit $(\$)$ in the three models (daily values)

The comparison between the three zones and one zone cases shows that the lack of coordination has two other effects. First, since there is less capacity available to generate electricity due to the higher reserve capacity requirement, consumers offer high downward reserve service, which implies high revenues for them (the DMS 
revenues in the coordinated case are almost null). Second, we observe a significant high value of the system reliability. Again, having a comparatively tighter power system results in more expensive flexibility services.

Finally, Table 10 gives the TSO's remuneration for each model that comes from the transmission system operation.

\begin{tabular}{ccc|ccc}
\hline \multicolumn{3}{c|}{ Three zones } & \multicolumn{3}{c}{ One zone } \\
\hline Model 1 & $\begin{array}{c}\text { Model 2 } \\
\text { (Expected) }\end{array}$ & $\begin{array}{c}\text { Model 3 } \\
\text { (Expected) }\end{array}$ & Model 1 & $\begin{array}{c}\text { Model 2 } \\
\text { (Expected) }\end{array}$ & $\begin{array}{c}\text { Model 3 } \\
\text { (Expected) }\end{array}$ \\
\hline 41,410 & 41,196 & 42,085 & 51,558 & 52,444 & 52,444 \\
\hline
\end{tabular}

Table 10: TSOs/CORESO's remuneration for each case (\$) (daily values)

The TSO's remuneration in the coordinated case is higher than in the not-coordinated case because there is more line congestion. Recall that with the not-coordinated case, the total amount of capacity that has to be destined to reserve increases because the minimum requirement of conventional reserve goes from 500 MW to 1200 MW. This implies a reduction of the generating capacity to generate electricity and, hence, less transmission congestion.

\section{Conclusions}

The non-dispatchability of renewable technologies introduces high uncertainty in the short-term operation. Reserve procurement represents a key aspect in power systems with high renewable capacity in order to ensure supply quality to consumers. Therefore, flexibility is mentioned as an emerging issue in order to tackle that variability and one may inquire about the impact of market design on the provision of flexibility. In this paper, three market designs are analyzed, which differ by the stage at which reserve capacity needs are determined. To analyze the performance of each market, three optimization models are proposed, which take into account the variability of the demand and the renewable power production in the real-time operation. On top of that, we analyze the impact of different degrees of coordination in reserve procurement on the market efficiency. The conclusions resulting from this study are as follows:

1) Model 1 results to be less efficient than the other two market designs under all assumptions. Due to the anticipativity of the reserve procurement, the TSO may procure a level of reserves that is much higher than the amount effectively needed.

2) Model 2, where the co-optimization of energy and reserve is operated by the TSO, is as efficient as Model 3 in the coordinated reserve procurement because it can substantially modify the outcome of the day-ahead energy market to procure reserve needs. When operating in a not-coordinated case, Model 2 becomes less efficient than Model 3 because of the imposition of cross-border day-ahead exchange limitations, but still more efficient than Model 1.

3) As expected, the co-optimized procurement of energy and reserve described by Model 3 is the most efficient market under all reserve procurement assumptions.

4) In all models, the not-coordinated reserve procurement based on multiple zones leads to higher total operating costs than considering the power system as a whole. In addition, involuntary unserved demand may happen in real time due to not being flexible enough to counteract the deviations between the day-ahead schedule and the real-time operation.

5) In the coordinated reserve procurement case, the market is mainly capacity driven since the flexibility is offered as a byproduct of existing capacity. In the not-coordinated case, ramping services play a significant role.

6) With respect to the operation of generating units, the study of the profits obtained by each technology shows that CCGT units mostly work as reserve units, increasing their profits in the not-coordinated case.

Additionally, these results provide a policy interpretation. Our case study is indeed conducted on a system that has ample conventional flexible capacity (2650 MW net capacity with $1855 \mathrm{MW} /$ hour of ramping capability for a peak demand of $3135 \mathrm{MW}$ ). Interestingly, the different models show that this physically comfortable market can behave quite differently for apparently minor modifications of market design. The not-coordinated case increases the operating costs, and the market with physically abundant flexible capacities suddenly becomes constrained in flexibility, leading to unserved demand in real time. This reminds of phenomena observed years ago with congestion phenomena that were supposed to be solved by counter-trading without modifying the commercial flows. However, similar effects are here observed with the reserve procurement and will probably lead to a higher impact with the increasing renewable production in an imperfect market design. In fact, the European Commission and system operators across the US are concerned about increasing the real-time coordination and that is why they are promoting modifications in the regulation. This paper provides a compelling outcome of the consequences of, first, having a certain market design, and second, how flexible the system is to provide the reserve capacity needed under high renewable production. 
In other words, our results give numbers to the outcome of the analysis conducted by Neuhoff et al. (2015) on reserve procurement that is "Reserve pooling across national borders can be an important benefit of European power market integration. In order to achieve this, pricing structure, product design, and timing need to be further aligned. A closer integration of reserve procurement with short-term energy markets will allow to capture benefits of co-optimization."

\section{Acknowledgement}

Giorgia Oggioni is grateful to the UniBS H\&W Project "Brescia 20-20-20" for the financial support. Ruth Domínguez is partly supported by Ministry of Economy and Competitiveness of Spain through CICYT project DPI2015-71280-R.

\section{References}

[(Andrade2017)] Andrade, J., Dong, Y., Baldick, R. (2017). Impact of Renewable Generation on Operational Reserves Requirements: When More Could be Less. White Paper UTEI/2016-11-2. Available at https: //energy.utexas.edu/files/2017/01/UTAustin_FCe_Ancillary_2017.pdf

[(Baldick2017)] Baldick, R. (2017). Seams, ancillary services and congestion management: US versus EU Electricity Markets. Available at https://medium.com/florence-school-of-regulation/seams-ancillaryservices-and-congestion-management-us-versus-eu-electricity-markets-ab9ad53a16df

[(Birge1997)] Birge, J. R., Louveaux, F. (1997). Introduction to Stochastic Programming. Springer-Verlag, New York, NY, 1997.

[(Bouffard2005)] Bouffard, F., Galiana, F.D., Conejo, A.J. (2005). Market-clearing with stochastic security - part I: formulation. IEEE Transaction on Power Systems, 20(4), 1818-1826.

[(Chen2014)] Chen, Y., Gribik, P. Gardner, J. (2017). Incorporating Post Zonal Reserve Deployment Transmission Constraints Into Energy and Ancillary Service Co-Optimization. IEEE Transaction on Power Systems, 29(2), 537-549.

[(Conejo2010)] Conejo, A. J., Carrión, M., Morales, J. M. (2010). Decision making under uncertainty in electricity markets. Springer, New York, NY.

[(Cramton2017)] Cramton, P. (2017). Electricity market design. Oxford Review of Economic Policy, 33(4), 589612.

[(CR17a)] Commission Regulation (2017a). Commission regulation (EU) 2017/1485 of 2 August 2017 establishing a guideline on electricity transmission system operation. Available at https://publications .europa.eu/en/ publication-detail/-/publication/d09a428c-8957-11e7-b5c6-01aa75ed71a1/language-en

[(CR17b)] Commission Regulation (2017b). Commission regulation (EU) 2017/2195 of 23 November 2017 establishing a guideline on electricity balancing. Available at https://publications.europa.eu/en/ publication-detail/-/publication/39af0e7c-d408-11e7-a5b9-01aa75ed71a1/language-en

[(Delikaraoglou2016)] Delikaraoglou, S., Morales, J. M., Pinson, P. (2016). Impact of inter- and intra-regional coordination in markets with a large renewable component. IEEE Transaction on Power Systems, 31(6), 5061-5070.

[(Doherty2005)] Doherty, R., O'Malley, M. (2005). A new approach to quantify reserve demand in systems with significant installed wind capacity. IEEE Transaction on Power Systems, 20(2), 587-595.

[(Dominguez2014)] Domínguez, R., Conejo, A.J., Carrión, M. (2014). Operation of a fully renewable electric energy system with CSP plants. Applied Energy, 119, 417-430.

[(Ela2011)] Ela, E., Milligan, M., Kirby B. (2011). Operating Reserves and Variable Generation, NREL Technical Report, NREL/TP-5500-51978.

[(EC2011)] European Commission (2011). Communication from the Commission to the European Parliament, the Council, The European Economic and Social Committee, the Committee of the Regions and the Committee of the Regions. Energy Roadmap 2050. European Commission, COM(2011) 885 final. Available at https: //eur-lex .europa.eu/legal-content/EN/TXT/PDF/?uri=CELEX : 52011DC0885\&from=EN

[(Fabbri2005)] Fabbri, A., Gomez San Roman, T., Rivier Abbad, J., Mendez Quezada, V. H. (2005). Assessment of the cost associated with wind generation prediction errors in a liberalized electricity market, IEEE Transaction on Power Systems, 20(3), 1440-1446. 
[(Flores2016)] Flores-Espino, F., Tian, T., Chernyakhovskiy, I. Megan, M., Miller M. (2016). Competitive Electricity Market Regulation in the United States: A Primer, NREL Technical Report, NREL/TP-6A20-67106.

[(Gebrekiros2015)] Gebrekiros, Y., Doorman, G., Jaehnert, S., Farahmand, H. (2015). Reserve procurement and transmission capacity reservation in the Northern European power market. Electrical Power and Energy Systems, 67, 546-559.

[(TERRE2017)] Nationalgrid, RED Electrica, REN, Rte, Terna, swissgrid (2017). Public consultation document for the design of the TERRE (Trans European Replacement Reserves Exchange). Executive summary RR Harmonized Balancing Area. Available at https://consultations.entsoe.eu/markets/publicconsultation-document-for-the-design-of-the/results/public-consultation-terre_exec_sum_ approval-package.pdf

[(weber)] Just, S., Weber, C. (2008). Pricing of reserves: Valuing system reserve capacity against spot prices in electricity markets. Energy Economics, 30(6), 3198-3221.

[(Lecadre2015)] Le Cadre, H., Papavasiliou, A., Smeers, Y. (2015). Wind farm portfolio optimization under network capacity constraints, European Journal of Operational Research, 247, 560-574.

[(Milligan2010)] Milligan et al. (2010). Operating reserves and wind power integration: an international comparison. NREL/CP-5500-49019. NREL. Available at https://www.nrel.gov/docs/fy11osti/49019.pdf

[(Morales2012)] Morales, J.M., Conejo, A. J., Liu, K., Zhong, J. (2012). Pricing electricity in pools with wind producers. IEEE Transaction on Power Systems, 27(3), 1366-1376.

[(Morales2017)] Morales, J.M., Pineda, S. (2017). On the inefficiency of the merit order in forward electricity markets with uncertain supply. European Journal of Operational Research, 261(2), 789-799.

[(neuhoff2015)] Neuhoff, K., Batlle C., Brunekreeft G., Vasilakos Konstantinidis C., Nabe C., Oggioni G., Rodilla P., Schwenen S., Siewierski T., Strbac, G. (2015). Flexible short-term power trading: Gathering experience in EU countries, DIW Berlin Discussion Paper, 1494.

[(newbery2016)] Newbery, D., Strbac, G., Viehoff, I. (2016). The benefits of integrating European electricity markets. Energy Policy, 94, 253-263.

[(Oggioni2014)] Oggioni, G., Murphy, F. H., Smeers, Y. (2014). Evaluating the impacts of priority dispatch in the European electricity market. Energy Economics, 42(0), 183-200.

[(ortega2009)] Ortega-Vazquez, M.A., Kirschen, D.S. (2009). Estimating the Spinning Reserve Requirements in Systems With Significant Wind Power Generation Penetration, IEEE Transaction on Power Systems, 24(1), 114-124.

[(anthony2011)] Papavasiliou, A., Oren, S.S., O'Neill, R.P. (2011). Reserve requirements for wind power integration: a scenario-based stochastic programming framework. IEEE Transaction on Power Systems, 26(4), $2197-2206$

[(anthony2017)] Papavasiliou, A., Smeers, Y. (2017). Remuneration of Flexibility using Operating Reserve Demand Curves: A Case Study of Belgium. Available at https://perso.uclouvain.be/anthony. papavasiliou/public_html/ORDCV12.pdf

[(pineda2016)] Pineda, S., Morales, J.M., Boomsma, T.K. (2016). Impact of forecast errors on expansion planning of power systems with a renewables target, European Journal of Operational Research, 248(3), 1113-1122.

[(Pritchard2010)] Pritchard, G., Zakeri, G., Philpott, A. (2010). A single-settlement, energy-only electric power market for unpredictable and intermittent participants. Operations Research, 58(4), 1210-1219.

[(hobbs17)] Van den Bergha, K., Hytowitzb, R. B., Bruninx, K., Delarue, E., Dhaeseleer, W., Hobbs B.F. (2017). Benefits of coordinating sizing, allocation and activation of reserves among market zones, Electric Power Systems Research, 43, 140-148.

[(fuller2007)] Wong, S., Fuller, D. (2007). Pricing energy and reserves using stochastic optimization in an alternative electricity market. IEEE Transaction on Power Systems, 22(2), 631-638.

[(Zavala2017)] Zavala, V. M., Kivaek, K., Anitescu, M., Birge, J. (2017). A stochastic electricity market clearing formulation with consistent pricing properties. Operations Research, 65(3), 557-576. 


\section{A Model notation}

This section lists the notation used in the mathematical formulations.

\section{Indices}

$f \quad$ Index of forecast scenarios in day ahead, running from 1 to $N_{F}$

$g \quad$ Index of generating units, running from 1 to $N_{G}$

$j \quad$ Index of demands, running from 1 to $N_{J}$

$l \quad$ Index of transmission lines, running from 1 to $N_{L}$

$n \quad$ Index of nodes, running from 1 to $N_{N}$

$s \quad$ Index of real time scenarios, running from 1 to $N_{S}$

$t \quad$ Index of time periods, running from 1 to $N_{T}$

$z \quad$ Index of network zones, running from 1 to $N_{Z}$

Sets

$D(j, n)$ Set of demands connected to node $n$

$G(g, n)$ Set of generating units connected to node $n$

$Z D(j, z) \quad$ Set of demands in zone $z$

$Z G(g, z) \quad$ Set of generating units in zone $z$

$\Omega^{\mathrm{Di} / \mathrm{St}} \quad$ Set of dispatchable/stochastic units

$\Omega_{f}$ Set of real-time scenarios generated from forecast scenario $f$

$\Omega^{\mathrm{nQ} / \mathrm{Q}}$ Set of not-qualified/qualified units

\section{Constants and parameters}

$B_{l} \quad$ Susceptance of the line $l$ (p.u.)

$C_{j}^{\mathrm{D}} \quad$ Cost of demand deviation (\$/MWh)

$C_{g t}^{\text {DDR/DUR }}$ Cost of deploying down/up reserves of generating unit $g$ in period $t(\$ / \mathrm{MWh})$

$C_{g}^{\mathrm{G}} \quad$ Generation cost of generating unit $g(\$ / \mathrm{MWh})$

$C^{\mathrm{US}}$ Cost of unserved demand $(\$ / \mathrm{MWh})$

$D_{j t}^{F} \quad$ Forecasted demand $j$ in period $t(\mathrm{MWh})$

$D_{j t s}^{\mathrm{RT}} \quad$ Actual demand $j$ in period $t$ and real time scenario $s(\mathrm{MWh})$

$K^{\mathrm{U} / \mathrm{D}}$ Security factor associated with upward/downward deviations of forecast errors (p.u.)

$P_{l}^{\mathrm{Lmax}}$ Capacity of the line $l(\mathrm{MW})$

$P_{g}^{\text {Gmax }}$ Capacity of generating unit $g(\mathrm{MW})$

$P_{g t s}^{\mathrm{RT}} \quad$ Actual power of stochastic generating unit $g$ in period $t$ and real time scenario $s$ (MWh)

$\operatorname{ramp}_{g}^{\text {do/up }}$ Down/up ramping limits of generating unit $g(\mathrm{MW} / \mathrm{h})$

$\Delta d_{j t}^{\max } \quad$ Maximum deviation of demand $j$ in period $t(\mathrm{MWh})$

$\sigma_{j t}^{\mathrm{D}} \quad$ Standard deviation of the forecasted error of demand $j$ in period $t(\mathrm{MW})$

$\sigma_{j t f}^{\mathrm{D}} \quad$ Standard deviation of the forecasted error of demand $j$ in period $t$ and forecast scenario $f(\mathrm{MW})$

$\sigma_{g t}^{\mathrm{St}}$ Standard deviation of the forecast error of stochastic production of unit $g$ in period $t$ (MW)

$\xi_{s} \quad$ Probability of real time scenario $s$ (p.u.)

$\xi_{f} \quad$ Probability of forecast scenario $f$ in day ahead (p.u.)

\section{Primal Variables}

$d_{j t}^{\mathrm{US}}$ Unserved power for demand $j$ in period $t$ in day ahead (MWh)

$d_{j t}^{\text {US }}{ }^{\text {TSO }}$ Unserved power for demand $j$ in period $t$ in day ahead in the TSO's Model 2 (MWh)

$d_{j t s}^{\mathrm{US}}$ Unserved power for demand $j$ in period $t$ and real time scenario $s$ (MWh)

$p_{g t}^{\mathrm{DA}} \quad$ Power generated in day ahead by generating unit $g$ in period $t(\mathrm{MWh})$ 
$p_{g t}^{\text {TSO }}$ Power re-schedule operated by TSO for dispatchable unit $g$ in period $t$ in the day ahead in Model $2(\mathrm{MWh})$

$p_{g t s}^{\mathrm{G}} \quad$ Power generated in real time by generating unit $g$ in period $t$ and scenario $s$ (MWh)

$p_{l t}^{\mathrm{LDA}}$ Power flow through line $l$ in period $t$ in day ahead (MWh)

$p_{l t}^{\mathrm{LTSO}}$ Power flow through line $l$ in period $t$ in day ahead in the TSO's Model 2 (MWh)

$p_{l t s}^{\mathrm{LRT}} \quad$ Power flow through line $l$ in period $t$ and real time scenario $s$ (MWh)

$R_{g t}^{\mathrm{D} / \mathrm{U}} \quad$ Down/up reserve scheduled by generating unit $g$ in period $t(\mathrm{MW})$

$R_{g t}^{\mathrm{C}} \quad$ Reserve for contingencies scheduled by generating unit $g$ in period $t$ (MW)

$r_{g t s}^{\mathrm{D} / \mathrm{U}}$ Down/up reserve deployed by generating unit $g$ in period $t$ and real time scenario $s$ (MWh)

$\Delta d_{j t s}^{\mathrm{D} / \mathrm{U}} \quad$ Down/up deviations by demand $j$ in period $t$ and real time scenario $s$ (MWh)

$\theta_{n t}^{\mathrm{DA}} \quad$ Voltage angle at node $n$ in period $t$ in day ahead $(\mathrm{rad})$

$\theta_{n t}^{\text {TSO }}$ Voltage angle at node $n$ in period $t$ in day ahead in the TSO's Model $2(\mathrm{rad})$

$\theta_{n t s}^{\mathrm{RT}} \quad$ Voltage angle at node $n$ in period $t$ and real time scenario $s(\mathrm{rad})$

Finally, we indicate in parentheses the corresponding dual variable next to each constraint.

\section{B Generating units' profits, consumers' benefit, and ISO's remu- neration}

In this section, we present the mathematical expressions used to compute the market agent (generators, consumers and TSO) profits. In particular, we use the equality between the primal and dual objective function values of our models to compute the agent profits. The primal problem represents the operation of the energy market and gives its cost. The dual problem represents the revenue accruing to the agents bringing assets to their market and the payment for electricity and its quality. Duality theory links the two, making possible to immediately evaluate the remuneration of the different resources (generating capacity, ramping services, DSM) from the profit realized by operating the system and selling its output at marginal cost.

We here concentrate on Model 1 by first reporting the objective function of the dual problem associated with (1)-(28) and then illustrating how the players' remuneration is determined. In particular, the terms composing the objective function (79) of the dual formulation are used to determine the generating units' profits, the consumers' benefit, and the ISO/TSO's remuneration depending on their economic interpretation. Agents' profits of Model 2 and Model 3 are derived in a similar way.

\section{B.1 Objective function of the dual formulation of Model 1}

The objective function of the dual formulation of Model 1 is as indicated in (79). 


$$
\begin{aligned}
& \operatorname{Max} \sum_{t=1}^{\mathrm{N}_{\mathrm{T}}} \sum_{j \in D(j, n)} \sum_{f=1}^{\mathrm{N}_{\mathrm{F}}} \xi_{f} D_{j t f}^{\mathrm{F}} \lambda_{n t f}^{\mathrm{DA}}-\sum_{t=1}^{\mathrm{N}_{\mathrm{T}}} \sum_{l=1}^{\mathrm{N}_{\mathrm{L}}} \sum_{f=1}^{\mathrm{N}_{\mathrm{F}}} \xi_{f} P_{l}^{\mathrm{Lmax}}\left(\rho_{l t f}^{\min }+\rho_{l t f}^{\max }\right)+ \\
& -\sum_{t=1}^{\mathrm{N}_{\mathrm{T}}} \sum_{n=1}^{\mathrm{N}_{\mathrm{N}}} \sum_{f=1}^{\mathrm{N}_{\mathrm{F}}} \xi_{f} \pi\left(\rho_{n t f}^{\pi, \min }+\rho_{n t f}^{\pi, \max }\right)-\sum_{t=1}^{\mathrm{N}_{\mathrm{T}}} \sum_{j=1}^{\mathrm{N}_{\mathrm{J}}} \sum_{f=1}^{\mathrm{N}_{\mathrm{F}}} \xi_{f} D_{j t f}^{\mathrm{F}} \psi_{j t f}-\sum_{t=1}^{\mathrm{N}_{\mathrm{T}}} \sum_{g \in \Omega^{\mathrm{St}}} \sum_{f=1}^{\mathrm{N}_{\mathrm{F}}} \xi_{f} P_{g t f}^{\mathrm{Gmax}} \mu_{g t f}+ \\
& -\sum_{t=1}^{\mathrm{N}_{\mathrm{T}}} \sum_{g \in \Omega^{\mathrm{n} \mathrm{Q}}} \sum_{f=1}^{\mathrm{N}_{\mathrm{F}}} \xi_{f} P_{g}^{\mathrm{Gmax}} \mu_{g t f}-\sum_{t=1}^{\mathrm{N}_{\mathrm{T}}} \sum_{g \in \Omega^{\mathrm{n} \mathrm{Q}}} \sum_{f=1}^{\mathrm{N}_{\mathrm{F}}} \xi_{f} \mathrm{ramp}^{\mathrm{up}} \mu_{g t f}^{\mathrm{rU}}-\sum_{t=1}^{\mathrm{N}_{\mathrm{T}}} \sum_{g \in \Omega^{\mathrm{n} Q}} \sum_{f=1}^{\mathrm{N}_{\mathrm{F}}} \xi_{f} \mathrm{ramp}^{\mathrm{do}} \mu_{g t f}^{\mathrm{rD}}+ \\
& -\sum_{t=1}^{\mathrm{N}_{\mathrm{T}}} \sum_{g \in \Omega^{\mathrm{Q}}} \sum_{f=1}^{\mathrm{N}_{\mathrm{F}}} \xi_{f} P_{g}^{\mathrm{Gmax}} \kappa_{g t f}^{\mathrm{U}}-\sum_{t=1}^{\mathrm{N}_{\mathrm{T}}} \sum_{g \in \Omega^{\mathrm{Q}}} \sum_{f=1}^{\mathrm{N}_{\mathrm{F}}} \xi_{f} \mathrm{ramp}^{\mathrm{up}} \kappa_{g t f}^{\mathrm{rU}}-\sum_{t=1}^{\mathrm{N}_{\mathrm{T}}} \sum_{g \in \Omega^{\mathrm{Q}}} \sum_{f=1}^{\mathrm{N}_{\mathrm{F}}} \xi_{f} \mathrm{ramp}^{\mathrm{do}} \kappa_{g t f}^{\mathrm{rD}}+ \\
& \left.+\sum_{t=1}^{\mathrm{N}_{\mathrm{T}}} \sum_{z=1}^{\mathrm{N}_{\mathrm{Z}}} \eta_{z t}^{\mathrm{D}}\left(K^{\mathrm{D}} \sqrt{\max _{f}\left\{\sum_{j \in Z D(j, z)}\left(\sigma_{j t f}^{\mathrm{D}}\right)^{2}\right\}+\max _{f}\left\{\sum_{g \in\left\{Z G(g, z), \Omega^{\mathrm{St}}\right\}}\left(\sigma_{g t f}^{\mathrm{St}}\right)^{2}\right.}\right\}\right) \\
& \left.+\sum_{t=1}^{\mathrm{N}_{\mathrm{T}}} \sum_{z=1}^{\mathrm{N}_{\mathrm{Z}}} \eta_{z t}^{\mathrm{U}}\left(K^{\mathrm{U}} \sqrt{\max _{f}\left\{\sum_{j \in Z D(j, z)}\left(\sigma_{j t f}^{\mathrm{D}}\right)^{2}\right\}+\max _{f}\left\{\sum_{g \in\left\{Z G(g, z), \Omega^{\mathrm{St}}\right\}}\left(\sigma_{g t f}^{\mathrm{St}}\right)^{2}\right.}\right\}\right) \\
& +\sum_{t=1}^{\mathrm{N}_{\mathrm{T}}} \sum_{z=1}^{\mathrm{N}_{\mathrm{Z}}} \eta_{z t}^{\mathrm{C}} \max _{g \in\left\{Z G(g, z), \Omega^{\mathrm{Di}}\right\}}\left\{P_{g}^{\mathrm{Gmax}}\right\}+ \\
& +\sum_{t=1}^{\mathrm{N}_{\mathrm{T}}} \sum_{j \in D(j, n)} \sum_{s=1}^{\mathrm{N}_{\mathrm{S}}}\left(D_{j t s}^{\mathrm{RT}}-D_{j t f}^{\mathrm{F}}\right) \lambda_{n t s}^{\mathrm{RT}}-\sum_{t=1}^{\mathrm{N}_{\mathrm{T}}} \sum_{l=1}^{\mathrm{N}_{\mathrm{L}}} \sum_{s=1}^{\mathrm{N}_{\mathrm{S}}} \xi_{s} P_{l}^{\mathrm{Lmax}}\left(\gamma_{l t s}^{\min }+\gamma_{l t s}^{\max }\right)+ \\
& -\sum_{t=1}^{\mathrm{N}_{\mathrm{T}}} \sum_{n=1}^{\mathrm{N}_{\mathrm{N}}} \sum_{s=1}^{\mathrm{N}_{\mathrm{S}}} \xi_{s_{f}} \pi\left(\gamma_{n t s_{f}}^{\pi, \min }+\gamma_{n s_{f}}^{\pi, \max }\right)-\sum_{t=1}^{\mathrm{N}_{\mathrm{T}}} \sum_{g \in \Omega^{\mathrm{St}}} \sum_{s=1}^{\mathrm{N}_{\mathrm{S}}} \xi_{s} P_{g t s}^{\mathrm{RT}} \tau_{g t s}+ \\
& -\sum_{t=1}^{\mathrm{N}_{\mathrm{T}}} \sum_{j=1}^{\mathrm{N}_{\mathrm{J}}} \sum_{s=1}^{\mathrm{N}_{\mathrm{S}}} \xi_{s} D_{j t s}^{\mathrm{RT}} \psi_{j t s}^{1}-\sum_{t=1}^{\mathrm{N}_{\mathrm{T}}} \sum_{j=1}^{\mathrm{N}_{\mathrm{J}}} \sum_{s=1}^{\mathrm{N}_{\mathrm{S}}} \xi_{s} \Delta d_{j t}^{\max }\left(\psi_{j t s}^{2}+\psi_{j t s}^{3}\right)
\end{aligned}
$$

Using duality theory, we group the terms of the dual objective function to define the profits as follows. We first consider the difference between the sum of the terms in "lambda" $\left(\lambda_{n t f}^{\mathrm{DA}}, \lambda_{n t f}^{\mathrm{RT}}\right)$ and in "eta" $\left(\eta_{z t f}^{\mathrm{D}}, \eta_{z t f}^{\mathrm{U}}, \eta_{z t f}^{\mathrm{C}}\right)$ and the value of the primal. The former are the consumers' payments for energy and reliability, the latter is the cost of operation. The difference between those two values is then the profit made by operating the fixed assets of the system, i.e. capacity and flexibility offered by generators and DSM capacity provided by the consumer. This expression states that the profit made by operating the fixed assets is equal to the payment to the owners of those fixed assets when valued at the respective dual prices. This relation is the standard principle of perfect competition in economics (which is our assumption of price taking agents): the rent paid to the providers of some resource (in this case the fixed assets) is equal to the gross profit made by these assets in the economy. The underpinning pricing interpretation is that capacity is paid at scarcity rent (the value of an additional unit of the asset) and the energy and reliability are priced at marginal cost. The rest of the reasoning consists of assigning the gross profit made on the energy and reliability to the different fixed assets (capacity, flexibility and demand side) according to the interpretation of the parameters of the right-hand side of the primal.

\section{B.2 Generating units}

The profits of the generating units are different according to unit type.

- Qualified units are remunerated for energy, reserves and ramping services according to the following expression:

$$
\operatorname{Profits}_{g}=\sum_{t=1}^{\mathrm{N}_{\mathrm{T}}} \sum_{f=1}^{\mathrm{N}_{\mathrm{F}}} \xi_{f}\left(P_{g}^{\mathrm{Gmax}} \kappa_{g t f}^{\mathrm{U}}+\operatorname{ramp}^{\mathrm{up}} \kappa_{g t f}^{\mathrm{rU}}+\operatorname{ramp}^{\mathrm{do}} \kappa_{g t f}^{\mathrm{rD}}\right) \quad \forall g \in \Omega^{\mathrm{Q}}
$$

The term $\sum_{t=1}^{\mathrm{N}_{\mathrm{T}}} \sum_{f=1}^{\mathrm{N}_{\mathrm{F}}} \xi_{f} P_{g}^{\mathrm{Gmax}} \kappa_{g t f}^{\mathrm{U}}$ corresponds to the expected remuneration of the unit from the capacity used to provide energy and reserve for the set of day-ahead forecasts. The term is the product between 
the unit generation capacity $P_{g}^{\mathrm{Gmax}}$ and the scarcity rent $\kappa_{g t f}^{\mathrm{U}}$, i.e. the dual variable of constraint (14). The terms $\sum_{t=1}^{\mathrm{N}_{\mathrm{T}}} \sum_{f=1}^{\mathrm{N}_{\mathrm{F}}}$ ramp $^{\mathrm{up}} \kappa_{g t f}^{\mathrm{rU}}$ and $\sum_{t=1}^{\mathrm{N}_{\mathrm{T}}} \sum_{f=1}^{\mathrm{N}_{\mathrm{F}}}$ ramp $^{\mathrm{do}} \kappa_{g t f}^{\mathrm{rD}}$ correspond to the expected remuneration of upward and downward ramping services (ramping capacity), respectively. Note that $\kappa_{g t f}^{\mathrm{rU}}$ and $\kappa_{g t f}^{\mathrm{rD}}$ are the economic values of upward/downward flexibility.

- Not-qualified units provide energy and ramping services. Their profits are stated as follows:

$$
\operatorname{Profits}_{g}=\sum_{t=1}^{\mathrm{N}_{\mathrm{T}}} \sum_{f=1}^{\mathrm{N}_{\mathrm{F}}} \xi_{f}\left(P_{g}^{\mathrm{Gmax}} \mu_{g t f}+\operatorname{ramp}^{\mathrm{up}} \mu_{g t f}^{\mathrm{rU}}+\operatorname{ramp}^{\mathrm{do}} \mu_{g t f}^{\mathrm{rD}}\right) \quad \forall g \in \Omega^{\mathrm{nQ}}
$$

where $\mu_{g t f}$ is the scarcity rent and corresponds to the dual variable of capacity constraint (11), while $\mu_{g t f}^{\text {rU }}$ and $\mu_{g t f}^{\mathrm{rD}}$ are the dual variables of the ramping constraints (12) and (13) respectively. As for the qualified units, the first term of the profits' formula defines the capacity remuneration, while the other two the ramping capacity remuneration.

- Wind/solar units are remunerated for the energy scheduled in the day-ahead market at the amount $\sum_{t=1}^{\mathrm{N}_{\mathrm{T}}} \sum_{f=1}^{\mathrm{N}_{\mathrm{F}}} \xi_{f} P_{g}^{\mathrm{Gmax}} \mu_{g t f}$, where $\mu_{g t f}$ is the dual variable of constraint (11) with $g \in \Omega^{\mathrm{St}}$. These revenues are then adjusted in real time by the term $\sum_{t=1}^{\mathrm{N}_{\mathrm{T}}} \sum_{s=1}^{\mathrm{N}_{\mathrm{S}}} \xi_{s} P_{g t s}^{\mathrm{RT}} \tau_{g t s}$ to account for the actual production and possible deviations from the day-ahead production. Note that $\tau_{g t s}$ is the dual variable of constraint $(25)$.

$$
\operatorname{Profits}_{g}=\sum_{t=1}^{\mathrm{N}_{\mathrm{T}}} \sum_{f=1}^{\mathrm{N}_{\mathrm{F}}} \xi_{f} P_{g}^{\mathrm{Gmax}} \mu_{g t f}+\sum_{t=1}^{\mathrm{N}_{\mathrm{T}}} \sum_{s=1}^{\mathrm{N}_{\mathrm{S}}} \xi_{s} P_{g t s}^{\mathrm{RT}} \tau_{g t s} \quad \forall g \in \Omega^{\mathrm{St}}
$$

\section{B.3 Consumers}

The consumers' group pays for: 1) the energy service and 2) the reliability provided by the scheduled reserves. Then, consumers' benefit is defined as their willingness to pay for the service minus what they effectively pay as stated in the following expression:

$$
\begin{aligned}
\text { Benefit } & =\sum_{t=1}^{\mathrm{N}_{\mathrm{T}}} \sum_{s=1}^{\mathrm{N}_{\mathrm{S}}} \xi_{s}\left(D_{j t s}^{\mathrm{RT}}-\Delta d_{j t}^{\mathrm{max}}\right) C^{U S}+\sum_{t=1}^{\mathrm{N}_{\mathrm{T}}} C_{j t}^{D} \Delta d_{j t}^{\mathrm{max}}+ \\
+ & \sum_{t=1}^{\mathrm{N}_{\mathrm{T}}} \sum_{s=1}^{\mathrm{N}_{\mathrm{S}}} \xi_{s} \Delta d_{j t}^{\max }\left(\psi_{j t s}^{2}+\psi_{j t s}^{3}\right)+ \\
& -\sum_{t=1}^{\mathrm{N}_{\mathrm{T}}} \sum_{f=1}^{\mathrm{N}_{\mathrm{F}}} \xi_{f} D_{j t f}^{\mathrm{F}} \lambda_{n t f}^{\mathrm{DA}}+\sum_{t=1}^{\mathrm{N}_{\mathrm{T}}} \sum_{f=1}^{\mathrm{N}_{\mathrm{F}}} \xi_{f} D_{j t f}^{\mathrm{F}} \psi_{j t f}-\sum_{t=1}^{\mathrm{N}_{\mathrm{T}}} \sum_{f=1}^{\mathrm{N}_{\mathrm{F}}} \sum_{s \in \Omega_{f}}\left(D_{j t s}^{\mathrm{RT}}-D_{j t f}^{\mathrm{F}}\right) \lambda_{n t s}^{\mathrm{RT}}+\sum_{t=1}^{\mathrm{N}_{\mathrm{T}}} \sum_{s=1}^{\mathrm{N}_{\mathrm{S}}} \xi_{s} D_{j t s}^{\mathrm{RT}} \psi_{j t s}^{1}+ \\
& -\sum_{t=1}^{\mathrm{N}_{\mathrm{T}}} \sum_{z=1}^{\mathrm{N}_{\mathrm{Z}}}\left(K^{\mathrm{D}} \sqrt{\max _{j \in Z D(j, z)}\left\{\sum_{j \in\left\{Z G(g, z), \Omega^{\mathrm{St}}\right\}}\left(\sigma_{g t f}^{\mathrm{St}}\right)^{2}\right\}}\right) \cdot \eta_{z t}^{D}+ \\
& -\sum_{t=1}^{\mathrm{N}_{\mathrm{T}}} \sum_{z=1}^{\mathrm{N}_{\mathrm{Z}}}\left(K^{\mathrm{U}} \sqrt{\max _{j \in}\left\{\sum_{j \in Z D(j, z)}\left(\sigma_{j t f}^{\mathrm{D}}\right)^{2}\right\}+\max _{f}\left\{\sum_{g \in\left\{Z G(g, z), \Omega^{\mathrm{St}}\right\}}\left(\sigma_{g t f}^{\mathrm{St}}\right)^{2}\right\}}\right) \cdot \eta_{z t}^{U}+ \\
& -\sum_{t=1}^{\mathrm{N}_{\mathrm{T}}} \sum_{z=1}^{\mathrm{N}_{\mathrm{Z}}}\left(\max _{g \in\left\{Z G(g, z), \Omega^{\mathrm{Di}}\right\}}\left\{P_{g}^{\mathrm{Gmax}}\right\}\right) \cdot \eta_{z t}^{C}, \quad \forall j \in D(j, n)
\end{aligned}
$$

1) The energy service is evaluated as the sum of the consumers' willingness to pay (in the usual sense of welfare economics when demand is fixed) and the value of the capacity offered for DSM services minus the cost of buying energy in day ahead and the net value of demand schedule deviations between day ahead and real time.

- Willingness to pay. The consumers' willingness to pay is expressed by the sum of the terms $\sum_{t=1}^{\mathrm{N}_{\mathrm{T}}} \sum_{s=1}^{\mathrm{N}_{\mathrm{S}}} \xi_{s}\left(D_{j t s}^{\mathrm{RT}}-\Delta d_{j t}^{\max }\right) C^{U S}$ and $\sum_{t=1}^{\mathrm{N}_{\mathrm{T}}} C_{j t}^{D} \Delta d_{j t}^{\max }$ appearing in the first line of (83). Since demand is exogenous, we identify the maximum electricity price that consumers are willing to pay in the value of the unexpected unserved demand that is $C^{U S}$. The price $C^{U S}$ is multiplied by the actual demand in real time $D_{j t s}^{\mathrm{RT}}$ corrected by the $\Delta d_{j t}^{\max }$ that is the maximum demand deviation operated by 
consumers in real time when providing DSM services. Due the different consumers' valuation between an interruption by the TSO and a DSM service, the term $\sum_{t=1}^{\mathrm{N}_{\mathrm{T}}} C_{j t}^{D} \Delta d_{j t}^{\max }$ is evaluated at $C_{j t}^{D}$ instead of $C^{U S}$. Finally, note that these two terms composing the willingness to pay do not directly appear in $(79)$.

- Remuneration of demand side management services. The consumers' remuneration for the demand adjustments in real time is quantified with the term in the second line of (83), where $\sum_{t=1}^{\mathrm{N}_{\mathrm{T}}} \sum_{s=1}^{\mathrm{N}_{\mathrm{S}}} \xi_{s} \Delta d_{j t}^{\max }\left(\psi_{j t s}^{2}+\psi_{j t s}^{3}\right)$ is the revenue for providing DSM service from the fixed amount $\Delta d_{j t}^{\text {max }}$. Following a reasoning similar to that applied for generating units, the remuneration for this services is obtained by multiplying $\Delta d_{j t}^{\max }$ by the dual variables associated with constraints (27) and (28), whose value identify the remuneration for DSM.

- Cost of purchasing energy in day ahead and deviation between day ahead and real time. The cost of buying energy in day ahead is defined by the term $\sum_{t=1}^{\mathrm{N}_{\mathrm{T}}} \sum_{f=1}^{\mathrm{N}_{\mathrm{F}}} \xi_{f} D_{j t f}^{\mathrm{F}} \lambda_{n t f}^{\mathrm{DA}}$, where $D_{j t f}^{\mathrm{F}}$ is the demand for forecast $f$ and $\lambda_{n t f}^{\mathrm{DA}}$ is the dual variable of the energy balance (5), which corresponds to the electricity price in day ahead. This represents a forward purchase because there is no delivery in day ahead. This amount is corrected by the term $\sum_{t=1}^{\mathrm{N}_{\mathrm{T}}} \sum_{f=1}^{\mathrm{N}_{\mathrm{F}}} \xi_{f} D_{j t f}^{\mathrm{F}} \psi_{j t f}$ from constraint (10) that imposes bounds on the unserved demand in day ahead. Note that $\psi_{j t f}$ has a positive value when (10) is binding, i.e., when the unserved demand in day ahead is equal to the demand forecast $D_{j t f}^{\mathrm{F}}$, which is a very extreme situation.

On the other side, the difference $\sum_{t=1}^{\mathrm{N}_{\mathrm{T}}} \sum_{f=1}^{\mathrm{N}_{\mathrm{F}}} \sum_{s \in \Omega_{f}}\left(D_{j t s}^{\mathrm{RT}}-D_{j t f}^{\mathrm{F}}\right) \lambda_{n t s}^{\mathrm{RT}}$ represents the evaluation of the deviation between the demand forecast $D_{j t f}^{\mathrm{F}}$ in day ahead and the actual demand $D_{j t s}^{\mathrm{RT}}$ in the real-time scenario associated with forecast $f$. In this case, $\lambda_{n t s}^{\mathrm{RT}}$ is the dual variable associated with the energy balance in real time (18) and defines the price settlement in real time. This amount is adjusted by $\sum_{t=1}^{\mathrm{N}_{\mathrm{T}}} \sum_{s=1}^{\mathrm{N}_{\mathrm{S}}} \xi_{s} D_{j t s}^{\mathrm{RT}} \psi_{j t s}^{1}$ that is obtained from constraint (26). As for the day ahead, this term describes the situation where the unserved demand in real time equals the real-time demand, corrected by possible deviation due to DSM. Again, this is a very exceptional situation.

2) The system reliability provided by reserves represents a cost that is charged to consumers in order to ensure the supply quality. The system reliability is evaluated by the last three terms of (83), where the amounts of reserve scheduled are multiplied by the dual variables $\eta_{z t}^{D}, \eta_{z t}^{U}$, and $\eta_{z t}^{C}$ of the respective reserve need constraints (2), (3), and (4). From an economic point of view, the dual $\eta_{z t}^{D}, \eta_{z t}^{U}$, and $\eta_{z t}^{C}$ identify the reserve value.

\section{B.4 The ISO}

The ISO's remuneration for the transmission services in day ahead and in real time that is defined as follows:

$$
\begin{aligned}
& \text { Remuneration }=\sum_{t=1}^{\mathrm{N}_{\mathrm{T}}} \sum_{l=1}^{\mathrm{N}_{\mathrm{L}}} \sum_{f=1}^{\mathrm{N}_{\mathrm{F}}} P_{l}^{\mathrm{Lmax}}\left(\rho_{l t f}^{\min }+\rho_{l t f}^{\max }\right)+\sum_{t=1}^{\mathrm{N}_{\mathrm{T}}} \sum_{n=1}^{\mathrm{N}_{\mathrm{N}}} \sum_{f=1}^{\mathrm{N}_{\mathrm{F}}} \pi\left(\rho_{n t f}^{\pi, \min }+\rho_{n t f}^{\pi, \max }\right)+ \\
& +\sum_{t=1}^{\mathrm{N}_{\mathrm{T}}} \sum_{l=1}^{\mathrm{N}_{\mathrm{L}}} \sum_{s=1}^{\mathrm{N}_{\mathrm{S}}} \xi_{s} P_{l}^{\mathrm{Lmax}}\left(\gamma_{l t s}^{\min }+\gamma_{l t s}^{\max }\right)+\sum_{t=1}^{\mathrm{N}_{\mathrm{T}}} \sum_{n=1}^{\mathrm{N}_{\mathrm{N}}} \sum_{s=1}^{\mathrm{N}_{\mathrm{S}}} \xi_{s} \pi\left(\gamma_{n t s}^{\pi, \min }+\gamma_{n t s}^{\pi, \max }\right)
\end{aligned}
$$

The ISO is remunerated for the use of its infrastructures. Its remuneration is obtained from the economic interpretation of the dual variables associated with the transmission constraints in day ahead and in real time. The first two terms of (84) refer to the day ahead and descend from constraints (7) and (8). These addends have the interpretation of transmission rights, but we do not discuss about them in this paper. The other two terms derive from constraints (20) and (21) and represent the remuneration of the network utilization in real time.

\section{Input data}

This section discusses the input data used for our case study. In particular, we consider a case study based on the IEEE 24-node Reliability Test System (RTS). ${ }^{18}$ The example comprises 17 demands and 38 transmission lines. The peak electricity demand is equal to $3135 \mathrm{MW}$ and the technical characteristics of the transmission system are taken from the original network provided in Reliability Test System Task Force. Figure 7 depicts a schema of the system and the distribution of the generation units. We assume that the availability factors of the

\footnotetext{
${ }^{18}$ Reliability Test System Task Force (1999). The IEEE reliability test system-1996, IEEE Transaction on Power Systems, 14(3),1010-1020.
} 
nuclear, coal and CCGT units are equal to 1 in order to simplify the discussion. Availability factors of wind and solar PV units are dependent on the scenarios. For space limitations, we do not provide those data.

\begin{tabular}{c|ccccc}
\hline Technology & $\begin{array}{c}\text { Unit } \\
\#\end{array}$ & $\begin{array}{c}\text { Node } \\
\#\end{array}$ & $\begin{array}{c}\text { Capacity } \\
\text { (MW) }\end{array}$ & $\begin{array}{c}\text { Ramping rate } \\
(\mathbf{p u})\end{array}$ & $\begin{array}{c}\text { Variable cost } \\
(\$ / \mathbf{M W h})\end{array}$ \\
\hline CCGT & 1 & 1 & 300 & 0.70 & 55 \\
CCGT & 2 & 2 & 250 & 0.70 & 56 \\
Coal & 3 & 7 & 300 & 0.70 & 35 \\
CCGT & 4 & 15 & 400 & 0.70 & 50 \\
Coal & 5 & 16 & 300 & 0.70 & 36 \\
CCGT & 6 & 21 & 300 & 0.70 & 52 \\
CCGT & 7 & 22 & 400 & 0.70 & 53 \\
CCGT & 8 & 23 & 400 & 0.70 & 12 \\
Nuclear & 9 & 12 & 500 & 0.05 & \\
\hline
\end{tabular}

Table 11: Characteristics of the dispatchable generation units

The generation system comprises one nuclear (N), two coal (CO), six CCGT, six wind (W), and four solar PV (PV) plants. Table 11 reports the data associated with the dispatchable units: location, variable cost, capacity, and ramping rates. Conventional units are run in merit order on the basis of their variable (fuel) costs. We consider that the ramping rate of both CCGT and coal units is equal to 0.7. We assume a "warm" start for coal units since they mostly work as base units. For CCGT units, even though it is technically possible for them to work at higher ramping rate, the intensive use of the flexibility of the CCGTs increases O\&M costs and reduces their technical life.

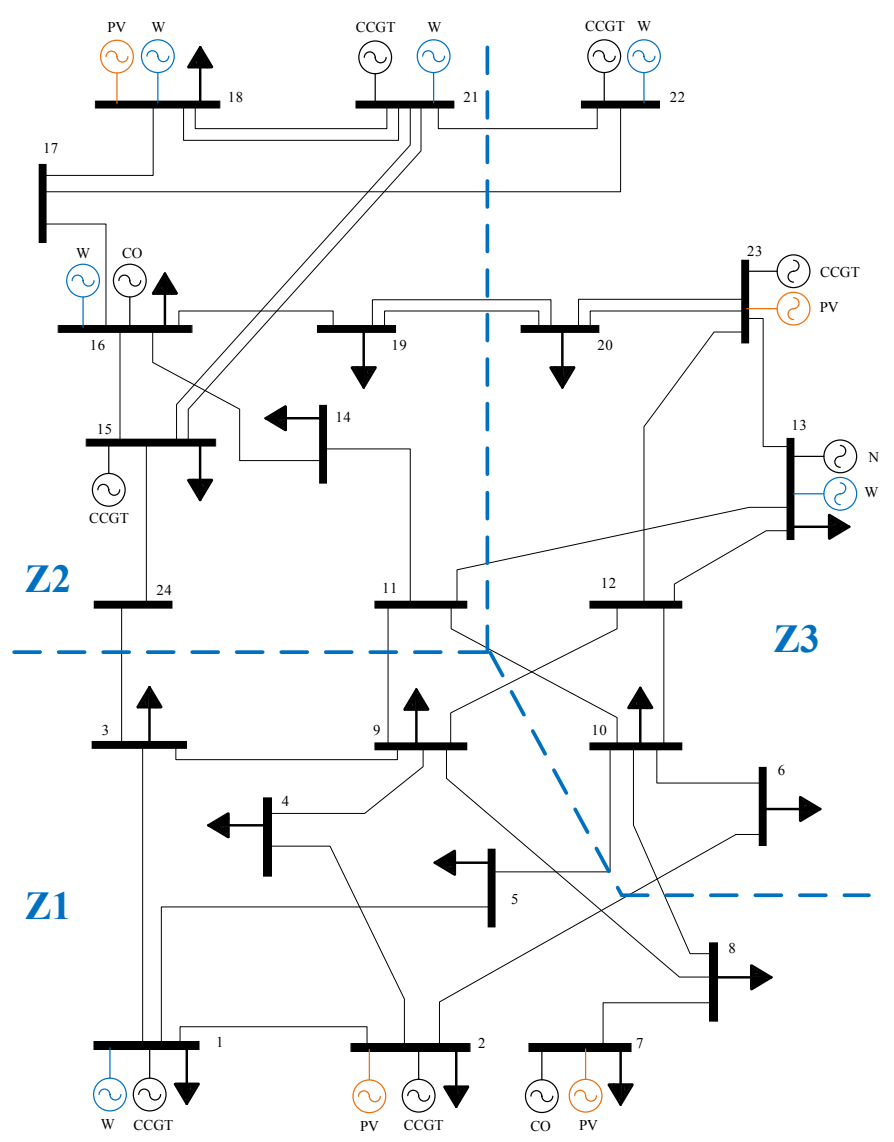

Figure 7: IEEE 24-node RTS.

The total generating capacity of the system is $6000 \mathrm{MW}$. Wind units have a capacity of $400 \mathrm{MW}$ and 300 MW, respectively. The four solar PV units have capacities of $200 \mathrm{MW}$ and $150 \mathrm{MW}$, respectively. Thus, the capacity per technology is distributed as follows: $2050 \mathrm{MW}(34 \%)$ of CCGT, $600 \mathrm{MW}$ (10\%) of coal, $500 \mathrm{MW}$ (8\%) of nuclear, $2100 \mathrm{MW}(35 \%)$ of wind, and $750 \mathrm{MW}$ (12.5\%) of solar PV. As customary in power systems 
with high share of stochastic units, the generation system is oversized with respect to the peak demand. In our case, the rate generating capacity/peak demand is 1.91 .

As explained in Section 2.1, the available renewable production in each node and at each hour is an uncertain parameter modeled through scenarios. The scenario generation process is detailed in D.

Consumer's demands are indicated by an arrow in Figure 7. Demand profiles can vary by zone. As for stochastic generation, nodal demand is exogenous and represented via scenarios as described in D. Unserved demand is priced at 10,000 $\$ / \mathrm{MWh}$, and up/down demand deviations in real time are priced between $89 \$ / \mathrm{MWh}$ and 93.2\$/MWh, being different among demands to avoid multiple solutions with the same objective function value.

All cases have been solved using CPLEX 12.6.3.0 (http://www.ilog.com/products/cplex/) under GAMS 24.6.1 on a Intel(R) Core(TM) $1.30 \mathrm{GHz}$ processor with 4 GB RAM.

\section{Scenario generation}

This section describes the scenario generation process. Our calibration is based on historical hourly data of the ERCOT power system. ${ }^{19}$ We rely on historical data of the demand, wind speed and solar irradiation from several locations in Texas available from NREL (2015) in the System Advisor Model. ${ }^{20}$ These data correspond to a typical meteorological year. We calibrate the short-term operating models on a Wednesday (D) in June.

Since in this analysis the uncertainty on the day-ahead and the balancing markets is taken into account, we generate demand, wind and solar scenarios for both stages. We refer to "forecast scenarios" to the scenarios considered in the day-ahead market, and to "real-time scenarios" to those for the balancing market. Three forecast scenarios and thirty real-time scenarios are generated.

First, the steps followed to generate the forecast scenarios are enumerated below:

- Demand level. Assuming that the variation of the hourly demand curve in a day of two consecutive weeks is comparatively low, we consider the actual demand level on the previous Wednesday (D-7) as the central prediction for the delivery day (D). Thus, we construct three forecast scenarios around this central prediction using a normal distribution with mean equal to the central prediction (D-7), and standard deviation equal to $5 \%$ Forecast scenarios are different among the three zones in which the network is divided.

- Solar power availability. To generate solar power scenarios, we assume low variability of the solar irradiation among consecutive days. We use historical data corresponding to the week before the day-ahead market (D-8), i.e., we consider a set of 168 values corresponding to the hours of a week. The procedure to generate the scenarios is as follows: first, the historical data are normalized using the maximum value among the considered set of hours, consequently, these values give an idea of the solar power availability in each hour; second, the mean over each hour of the day is computed; third, the solar power availability scenarios are generated using a normal distribution with mean equal to the mean value of the historical data and a standard deviation of $5 \%$ of the mean value. Three forecast scenarios per zone are generated.

- Wind power availability. The wind forecast scenarios are generated as follows:

- We use historical data of the hourly wind speed of four locations corresponding to the week before (D-8) of the day-ahead market.

- The next step consists on characterize the wind speed in each location. To do that, first we analyze whether there is spatial correlation among the wind speed data. If there is spatial correlation among the locations, it will be taken into account further to generate the scenarios.

- Second, we use computational tools to obtain the parameters that define the Weibull distribution of the wind speed in each location, and then the Weibull cumulative distribution function. The resulting values are fitted into a normal distribution using the inverse cumulative distribution function.

- Third, an ARMA model is generated per location using the data obtained in the previous step.

- Fourth, we generate forecast error scenarios assuming a white noise distribution. Then, we use the ARMA model and the forecast error scenarios to generate the forecast wind scenarios. At this step, we obtain scenarios of the wind speed in each location for 24 hours.

- Finally, we apply the power curve of a wind turbine ${ }^{21}$ to transform the $\mathrm{m} / \mathrm{s}$ to MW. Then, the production of a wind turbine is multiplied by the number of turbines comprising the wind farm to obtain the total wind power available.

\footnotetext{
${ }^{19}$ The Electric Reliability Council of Texas http://www.ercot.com

${ }^{20}$ NREL-National Renewable Energy Laboratory (2015). Alliance for Sustainable Energy (Alliance), Department of Energy (DOE). Solar Advisor Model version 2015.1.30.

${ }^{21}$ Source: Danish Wind Industry Association, Wind Turbine Power Calculator[Online]. Available at http://www. windpower.org
} 
Note that the demand level and the solar power availability are in per unit. Therefore, to obtain the consumption in MWh in each node, we multiply the per unit factor by the maximum demand level in each node (these data are available in Reliability Test System Task Force, 1999). Similarly, the available solar power results from multiplying the availability factor in each scenario times the unit capacity.

Second, real-time scenarios for the balancing market are generated regarding the forecast scenarios. Ten real-time scenarios are generated for each forecast scenario. The procedure is described bellow.

- Demand level and solar power availability. Real-time scenarios of both demand level and solar power availability are generated in the same way. Real-time scenarios are generated using a normal distribution with mean equal to the corresponding value of the forecast scenario in each hour, and a standard deviation equal to $1 \%$ of mean value. Consequently, 30 different values per hour ( 3 forecast scenarios times 10 realtime scenarios) are obtained. As with the forecast scenarios, the per unit values are transformed into actual quantities.

- Wind power availability. To generate real-time wind power scenarios, we apply the approach described in Pineda and Morales (2016), which is based on assuming that the forecast error follows a beta distribution with zero mean and standard deviation depending on the wind power prediction (Fabbri et al., 2005). Therefore, we express the standard deviation of the forecast error distributions of the real-time scenarios as a linear function of the value of the corresponding forecast scenario in each hour. The resulting real-time scenarios provide the wind power available in each hour and each node of the network. 